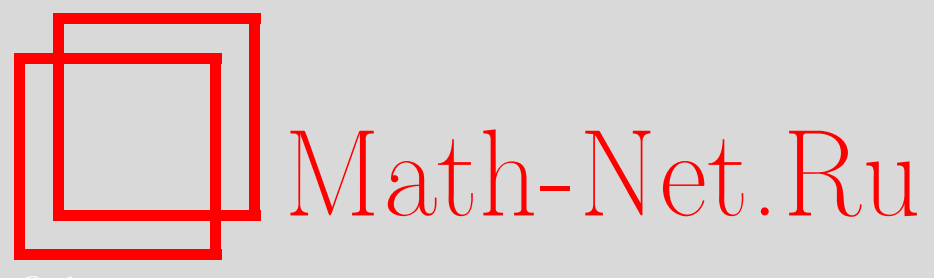

С. Д. Глызин, А. Ю. Колесов, Н. Х. Розов, Явление буферности в кольцевых цепочках однонаправленно связанных генераторов, Изв. РАН. Сер. матем., 2014, том 78, выпуск 4, 73-108

DOI: https://doi.org/10.4213/im8102

Использование Общероссийского математического портала Math-Net.Ru подразумевает, что вы прочитали и согласны с пользовательским соглашением http://www . mathnet.ru/rus/agreement

Параметры загрузки:

IP : 54.166 .219 .16

26 апреля 2023 г., 14:01:29

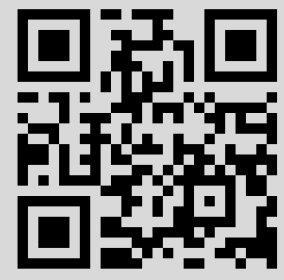


УДК 517.926

\author{
С. Д. Глызин, А. Ю. Колесов, Н. Х. Розов
}

\title{
Явление буферности в кольцевых цепочках однонаправленно связанных генераторов
}

\begin{abstract}
Вводится в рассмотрение новый объект - система обыкновенных дифференциальных уравнений, являющаяся математической моделью кольцевой цепочки однонаправленно связанных RCL-генераторов. Для исследования ее периодических решений типа бегущих волн используются некоторые специальные приемы, сводящие проблемы существования и устойчивости циклов к анализу вспомогательных уравнений с запаздыванием. На этом пути устанавливается, что при увеличении числа звеньев цепочки количество сосуществующих в ней устойчивых бегущих волн неограниченно растет, т. е. имеет место известное явление буферности.
\end{abstract}

Библиография: 12 наименований.

Ключевые слова: цепочка однонаправленно связанных генераторов, бегущая волна, асимптотика, устойчивость, явление буферности.

DOI: $10.4213 / \mathrm{im} 8102$

\section{§ 1. Постановка задачи и методы исследования}

1.1. Вывод математической модели. Общие принципы проектирования кольцевых цепочек однонаправленно связанных генераторов уместно проиллюстрировать на конкретном примере. В качестве такового возьмем систему RCL-генераторов и приведем вывод соответствующей математической модели.

Начальный этап построения любой кольцевой системы заключается в выборе так называемого парциального генератора, который в дальнейшем будет использован в качестве звена в соответствующей цепочке. В интересующем нас случае этот выбор осуществляется следующим образом.

Как и в работе [1], рассмотрим цепочку из $N$ последовательно соединенных RCL-контуров, к левому концу которой присоединен нелинейный элемент с монотонной вольт-амперной характеристикой $i=f(u)$ лампового типа (рис. 1,2$)$. Как известно [2], для токов $i_{n}=i_{n}(t)$ и напряжений $u_{n}=u_{n}(t)$ в такой цепочке справедлива система уравнений

$$
C \frac{d u_{n}}{d t}+i_{n+1}=i_{n}, \quad R i_{n}+L \frac{d i_{n}}{d t}=u_{n-1}-u_{n}, \quad n=1, \ldots, N,
$$

которую, естественно, следует дополнить уравнениями для $i_{N+1}$ и $u_{0}$. В данном случае $i_{N+1}$ - это ток на входе нелинейного элемента, выражающийся через входное напряжение $u_{N}$ посредством равенства

$$
i_{N+1}=\frac{u_{N}}{R_{\text {in }}},
$$

Работа выполнена при финансовой поддержке РФФИ (гранты № 11-01-00384_a, № 12-01-00155_a).

(C) С. Д. Глызин, А. Ю. Колесов, Н. Х. Розов, 2014 
где $R_{\text {in }}$ - входное сопротивление усилителя. В качестве переменной $u_{0}$ следует взять напряжение в узле $S$ (см. рис. 1$)$. Первый закон Кирхгофа и закон Ома для данного узла приводят к равенствам $i_{0}=f\left(u_{N}\right)+i_{1}, i_{0}=\left(E-u_{0}\right) / R_{\text {out }}$, где $i_{0}$ - ток, текущий через выходное сопротивление усилителя $R_{\text {out }}$ (см. рис. 1$)$.

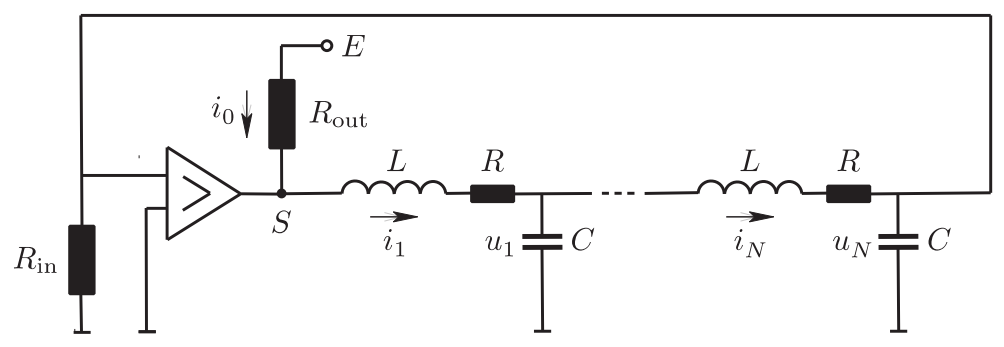

Рис. 1

Отсюда, в свою очередь, получаем требуемое уравнение для $u_{0}$, имеющее вид

$$
u_{0}+R_{\text {out }} f\left(u_{N}\right)+R_{\text {out }} i_{1}=E .
$$

Из приведенных физических соображений следует, что математической моделью рассматриваемого генератора служит система (1.1)-(1.3). В дальнейшем нам потребуется эта система в простейшем ее варианте, когда низкочастотный фильтр состоит только из одного RCL-контура, а усилитель является идеальным, т. е. $R_{\text {in }}=\infty$. Учитывая в $(1.1)-(1.3)$ равенства $N=1, R_{\text {in }}=\infty, u_{1}=u$, $i_{1}=i$ и исключая переменную $i$, в интересующем нас случае приходим к уравнению

$$
L C \ddot{u}+\left(R+R_{\text {out }}\right) C \dot{u}+u=E-R_{\text {out }} f(u) ;
$$

здесь и всюду далее точкой обозначается дифференцирование по $t$.

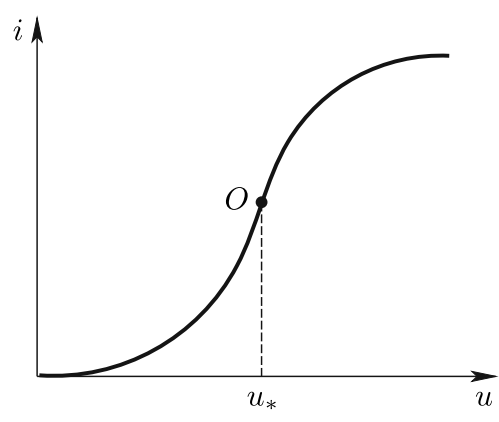

Рис. 2

Для удобства последующего анализа выполним в (1.4) замены $t / \sqrt{L C} \rightarrow t$, $u=u_{*}+x$, где $u_{*}$ - единственное (в силу монотонности $\left.f(u)\right)$ решение уравнения $u+R_{\text {out }} f(u)=E$, определяющее положение рабочей точки $O$ на нелинейной характеристике $i=f(u)$ (см. рис. 2). В результате получаем уравнение

$$
\ddot{x}+2 \mu \dot{x}+x=g(x),
$$


в котором $2 \mu=\left(R+R_{\text {out }}\right) \sqrt{C / L}, g(x)=E-R_{\text {out }} f\left(u_{*}+x\right)-u_{*}$. Предположим, далее, что фигурирующая в (1.5) функция $g(x)$ записывается в виде $g(x)=F(x / \varepsilon)$, где $1 / \varepsilon>0$ - коэффициент усиления нелинейного элемента, а функция $F(x) \in C^{\infty}(\mathbb{R})$ не зависит от $\varepsilon$ и обладает следующими свойствами (обусловленными характером поведения нелинейности $i=f(u)$ ):

$$
\begin{gathered}
F(0)=0, \quad F^{\prime}(x)<0 \quad \forall x \in \mathbb{R}, \\
F(x)=\left\{\begin{array}{lll}
1+\sum_{s=1}^{\infty} \frac{c_{s}^{-}}{x^{s}} & \text { при } & x \rightarrow-\infty, \\
-1+\sum_{s=1}^{\infty} \frac{c_{s}^{+}}{x^{s}} & \text { при } & x \rightarrow+\infty .
\end{array}\right.
\end{gathered}
$$

Предполагаем также, что представления (1.7) остаются в силе при дифференцировании по $x$ любое число раз. Типичными представителями таких функций служат

$$
F(x)=-\frac{2}{\pi} \operatorname{arctg} x, \quad F(x)=-\frac{x}{\sqrt{x^{2}+1}}, \quad F(x)=-\operatorname{th} x
$$

(в последнем случае все коэффициенты $c_{s}^{ \pm}, s \geqslant 1$, равны нулю).

Описанное выше одноконтурное устройство в принципе уже не является генератором, а представляет собой так называемый пассивный элемент. Действительно, автоколебания в нем отсутствуют, поскольку в силу условий (1.6) нулевое состояние равновесия уравнения (1.5) глобально экспоненциально устойчиво. Вернуть же данному устройству автоколебательные свойства можно по крайней мере двумя способами. Первый из них, предложенный в статье [1], состоит в добавлении запаздывания в цепь обратной связи, а второй заключается в следующем. Рассмотрим $m \geqslant 2$ идентичных экземпляров пассивных элементов указанного типа и объединим их в кольцо посредством однонаправленных связей. При $m=2$ соответствующая блок-схема представлена на рис. 3 , где требуемые связи показаны стрелками (по поводу их физической реализации см., например, [3], [4]).

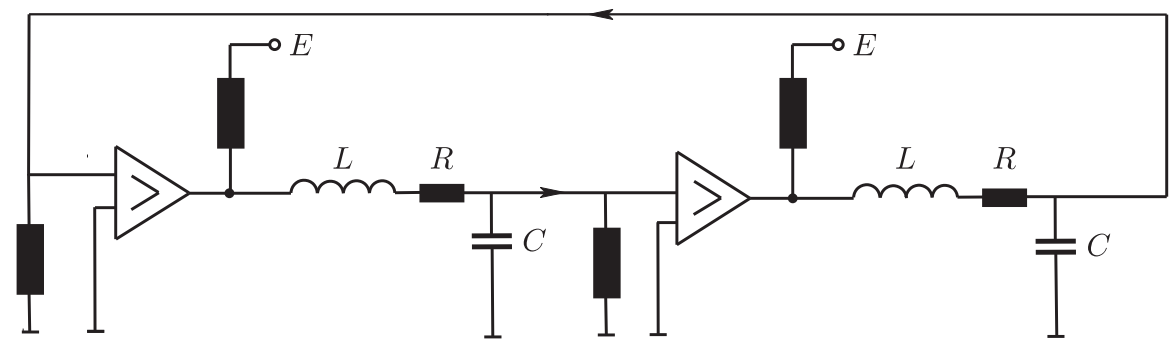

Рис. 3

Математически переход от пассивного RCL-контура к соответствующей кольцевой цепочке означает переход от уравнения (1.5) к системе

$$
\ddot{x}_{j}+2 \mu \dot{x}_{j}+x_{j}=F\left(\frac{x_{j-1}}{\varepsilon}\right), \quad j=1, \ldots, m, \quad x_{0}=x_{m},
$$


которая, собственно, и является объектом дальнейшего анализа. Точнее, нас будут интересовать вопросы о существовании и устойчивости ее бегущих волн, т. е. периодических решений вида

$$
x_{j}=x(t+(j-1) \Delta), \quad j=1, \ldots, m, \quad \Delta=\text { const }>0,
$$

в случае, когда $\varepsilon, \mu>0$ - независимые малые параметры.

Предложенная выше система (1.8) служит одним из типовых примеров кольцевых цепочек однонаправленно связанных генераторов. Рассмотрение таких цепочек актуально по крайней мере по двум причинам. Во-первых, как уже было сказано выше, данная связь весьма популярна в радиофизике [3], [4], поскольку допускает простую техническую реализацию. Во-вторых, использование этой связи в ряде случаев гарантирует сосуществование достаточно большого числа устойчивых периодических движений типа бегущих волн, т. е. реализуемость явления буферности. Последнее может быть применено, например, для моделирования функционирования ассоциативной памяти в нейронных сетях (по этому поводу см. соответствующее место в нашей предыдущей статье [5]). В частности, одной из возможных моделей ассоциативной памяти является устройство, блок-схема которого приведена на рис. 3.

Следует добавить, что бегущие волны представляют собой наиболее естественные рабочие режимы кольцевых однонаправленно связанных генераторов. Однако в рассматриваемых системах возможны и более сложные колебания. В качестве примера сошлемся на статью [6], в которой с помощью численного анализа показана реализуемость хаотического автоколебательного режима в кольце из трех однонаправленно связанных генераторов. Необходимо также отметить целую серию работ (см., например, [7]-[9]), посвященных проблеме перехода к хаосу в однонаправленно связанных цепочках осцилляторов при подходящем увеличении числа звеньев.

1.2. Общая схема исследования. Поскольку используемые нами методы анализа бегущих волн достаточно универсальны, имеет смысл описать их в самой общей ситуации, т. е. для произвольной кольцевой системы

$$
\dot{x}_{j}=f\left(x_{j}, x_{j-1}\right), \quad j=1, \ldots, m, \quad x_{0}=x_{m},
$$

где $m \geqslant 2, x_{j}=x_{j}(t) \in \mathbb{R}^{n}$, а вектор-функция $f(x, y)$ со значениями в $\mathbb{R}^{n}$ бесконечно дифференцируема по $(x, y) \in \mathbb{R}^{n} \times \mathbb{R}^{n}$.

Для отыскания циклов системы (1.10) вида (1.9) введем в рассмотрение вспомогательное уравнение с запаздыванием

$$
\dot{x}=f(x, x(t-\Delta)),
$$

где $x=x(t) \in \mathbb{R}^{n}$, и будем считать, что на некотором интервале $\left(\Delta_{1}, \Delta_{2}\right) \subset$ $(0,+\infty)$ изменения параметра $\Delta$ оно допускает нетривиальное (отличное от константы) периодическое решение $x=x_{*}(t, \Delta)$ периода $T_{*}=T_{*}(\Delta)>0$. В этом случае справедливо следующее утверждение.

Лемма 1.1. Предположим, что найдется такое натуральное $k$, при котором уравнение

$$
T_{*}(\Delta)=\frac{m \Delta}{k}
$$


имеет корень $\Delta=\Delta_{(k)} \in\left(\Delta_{1}, \Delta_{2}\right)$. Тогда в исходной системе (1.10) данному корню соответствует иикл (бегущая волна)

$$
C_{k}: x_{j}=x_{(k)}\left(t+(j-1) \Delta_{(k)}\right), \quad j=1, \ldots, m,
$$

периода $T_{(k)}=m \Delta_{(k)} / k$, дде $x_{(k)}(t)=\left.x_{*}(t, \Delta)\right|_{\Delta=\Delta_{(k)}}$.

ДокАЗАТЕЛЬСтво. Заметим, что, поскольку все функции

$$
x_{j}(t)=x_{(k)}\left(t+(j-1) \Delta_{(k)}\right), \quad j=1, \ldots, m,
$$

являются решениями одного и того же уравнения (1.11) при $\Delta=\Delta_{(k)}$, выполняются равенства

$$
\dot{x}_{j}(t)=f\left(x_{j}(t), x_{j}\left(t-\Delta_{(k)}\right)\right), \quad j=1, \ldots, m .
$$

Далее, учтем в (1.15) вытекающие из (1.14) соотношения

$$
\begin{gathered}
x_{j}\left(t-\Delta_{(k)}\right)=x_{j-1}(t), \quad j=2, \ldots, m, \\
x_{1}\left(t-\Delta_{(k)}\right)=x_{(k)}\left(t-\Delta_{(k)}\right)=x_{(k)}\left(t-\Delta_{(k)}+k T_{(k)}\right) \\
=x_{(k)}\left(t+(m-1) \Delta_{(k)}\right)=x_{m}(t) .
\end{gathered}
$$

В результате убеждаемся в том, что функции (1.14) удовлетворяют исходной системе (1.10). Лемма доказана.

Вопрос об устойчивости цикла (1.13) сводится, очевидно, к вопросу о расположении мультипликаторов линейной системы

$$
\dot{h}_{j}=A_{(k)}\left(t+(j-1) \Delta_{(k)}\right) h_{j}+B_{(k)}\left(t+(j-1) \Delta_{(k)}\right) h_{j-1}, \quad j=1, \ldots, m,
$$

где $h_{j}=h_{j}(t) \in \mathbb{R}^{n}, h_{0}=h_{m}$, а матрицы $A_{(k)}(t), B_{(k)}(t)$ задаются равенствами

$$
A_{(k)}(t)=f_{x}^{\prime}\left(x_{(k)}(t), x_{(k)}\left(t-\Delta_{(k)}\right)\right), \quad B_{(k)}(t)=f_{y}^{\prime}\left(x_{(k)}(t), x_{(k)}\left(t-\Delta_{(k)}\right)\right) .
$$

Наряду с (1.16) в дальнейшем нам понадобится вспомогательное линейное уравнение

$$
\dot{h}=A_{(k)}(t) h+\varkappa B_{(k)}(t) h\left(t-\Delta_{(k)}\right),
$$

где $h(t) \in \mathbb{C}^{n}, \varkappa-$ произвольный комплексный параметр. Точнее, нас будут интересовать его мультипликаторы $\nu_{s}(\varkappa), s=1,2, \ldots$, занумерованные в порядке убывания модулей.

Поясним смысл термина "мультипликатор" применительно к уравнению с запаздыванием (1.17). В связи с этим рассмотрим банахово пространство $E=C\left(\left[-\Delta_{(k)}, 0\right] ; \mathbb{C}^{n}\right)$ непрерывных при $-\Delta_{(k)} \leqslant t \leqslant 0$ вектор-функций $h^{0}(t)=\left(h_{1}^{0}(t), \ldots, h_{n}^{0}(t)\right)$ с нормой

$$
\left\|h^{0}\right\|_{E}=\max _{1 \leqslant l \leqslant n} \max _{-\Delta_{(k)} \leqslant t \leqslant 0}\left|h_{l}^{0}(t)\right| .
$$

Далее, оператором монодромии уравнения (1.17) назовем линейный ограниченный оператор $V: E \rightarrow E$, действующий на произвольную функцию $h^{0}(t) \in E$ по правилу

$$
V h^{0}=h\left(t+\frac{m \Delta_{(k)}}{k}\right), \quad-\Delta_{(k)} \leqslant t \leqslant 0,
$$


где $h(t)$ - решение уравнения (1.17) на отрезке времени $0 \leqslant t \leqslant m \Delta_{(k)} / k$ с начальной функцией $h^{0}(t),-\Delta_{(k)} \leqslant t \leqslant 0$. Отметим, что спектр этого оператора заведомо дискретен, так как некоторая его степень компактна (в случае $m / k \geqslant 1$ компактен и сам оператор $V$ ). Мультипликаторами уравнения (1.17) по аналогии со случаем обыкновенных дифференциальных уравнений будем называть собственные значения оператора (1.18).

Вопрос о связи между мультипликаторами систем (1.16) и (1.17) изложим сначала на неформальном уровне строгости. Как оказывается, для отыскания мультипликаторов бегущей волны (1.13) можно использовать так называемый метод подстройки по параметру $\varkappa$ в определенном смысле аналогичный процессу настройки радиоприемника на нужную станцию. Суть этого метода такова.

Выберем из счетного семейства мультипликаторов системы (1.17) любой мультипликатор $\nu(\varkappa)$ и рассмотрим отвечающее ему уравнение $(\nu(\varkappa))^{k}=\varkappa^{m}$. Как будет показано ниже, каждому корню $\varkappa=\varkappa_{0} \neq 0$ этого уравнения соответствует мультипликатор системы (1.16), задающийся равенством $\nu=\nu\left(\varkappa_{0}\right)$. Верно и обратное утверждение: на любой мультипликатор $\nu$ цикла (1.13) мож-

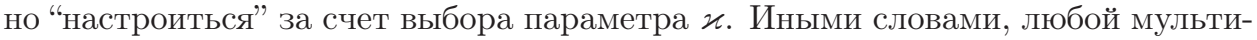
пликатор $\nu$ данного цикла допускает представление $\nu=\nu\left(\varkappa_{0}\right)$, где $\nu(\varkappa)-$ один из мультипликаторов вспомогательной системы (1.17), а $\varkappa_{0}$ - корень соответствующего уравнения $(\nu(\varkappa))^{k}=\varkappa^{m}$. Таким образом, набор всех ненулевых корней указанных уравнений можно рассматривать как совокупность "радиочастот", на которых работают интересующие нас станции. Сам же факт "настройки на станцию" соответствует конкретному выбору одного из этих корней и получению на выходе соответствующего мультипликатора $\nu=\nu\left(\varkappa_{0}\right)$ системы (1.16).

Строгий смысл методу подстройки придает следующее утверждение.

ЛЕмма 1.2. Каждый мулътипликатор у системы (1.16) допускает представление

$$
\nu=\varkappa^{m / k}
$$

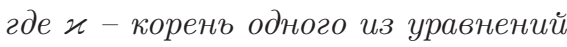

$$
\left[\nu_{s}(\varkappa)\right]^{k}=\varkappa^{m}, \quad s \in \mathbb{N} .
$$

И обратно, если при некотором $s=s_{0}$ уравнение (1.20) имеет ненулевой корень $\varkappa=\varkappa_{0}$, то для исходной системы (1.16) существует мультипликаmop $\nu=\nu_{s_{0}}\left(\varkappa_{0}\right)$.

ДокАЗАТЕЛЬСтво. Фиксируем любой мультипликатор $\nu=\rho \exp (i \varphi)$, где $\rho>0,0 \leqslant \varphi<2 \pi$, системы (1.16) и предположим, что он является простым. В этом случае ему отвечает единственное (с точностью до множителя) решение Ляпунова-Флоке, т. е. решение вида

$$
\begin{gathered}
h_{j}=\exp (\alpha t) h_{*, j}(t), \quad h_{*, j}(t) \in \mathbb{C}^{n}, \quad h_{*, j}\left(t+\frac{m \Delta_{(k)}}{k}\right) \equiv h_{j, *}(t), \quad j=1, \ldots, m, \\
\alpha=\frac{k}{m \Delta_{(k)}}(\ln \rho+i \varphi) .
\end{gathered}
$$


Отметим, далее, что, поскольку система (1.16) инвариантна относительно замен

$$
t-\Delta_{(k)} \rightarrow t, \quad h_{j-1} \rightarrow h_{j}, \quad j=1, \ldots, m,
$$

под действием этих замен решение (1.21) (в силу его единственности) перейдет в решение

$$
h_{j}=\lambda \exp \left(\alpha\left(t+\Delta_{(k)}\right)\right) h_{*, j}(t), \quad j=1, \ldots, m,
$$

где $\lambda \neq 0$ - некоторая комплексная постоянная. Таким образом, имеет место равенство

$$
\Lambda h_{*}\left(t+\Delta_{(k)}\right)=\lambda h_{*}(t),
$$

где $h_{*}(t)=\operatorname{colon}\left(h_{*, 1}(t), \ldots, h_{*, m}(t)\right)$, а элементами $(n m \times n m)$-матрицы

$$
\Lambda=\left(\begin{array}{cccccc}
0 & 0 & 0 & \ldots & 0 & I \\
I & 0 & 0 & \ldots & 0 & 0 \\
0 & I & 0 & \ldots & 0 & 0 \\
\ldots & \ldots & \ldots & \ldots & \ldots & \ldots \\
\ldots & \ldots & \ldots & \ldots & \ldots & \ldots \\
0 & 0 & 0 & \ldots & I & 0
\end{array}\right)
$$

являются нулевые и единичные $(n \times n)$-матрицы.

Из установленного выше соотношения (1.23) следует, что

$$
h_{*, m-j}(t)=\lambda^{j+1} h_{*, 1}\left(t-(j+1) \Delta_{(k)}\right), \quad j=0,1, \ldots, m-2, \quad \lambda^{m}=1 .
$$

Компонента $h_{*, 1}(t)$ в силу (1.16) является решением линейного неоднородного уравнения

$$
\dot{h}=-\alpha h+A_{(k)}(t) h+B_{(k)}(t) h_{*, m}(t) .
$$

Поскольку функция $h_{*, m}(t)$, в свою очередь, выражается через $h_{*, 1}(t)$ посредством равенства $h_{*, m}(t)=\lambda h_{*, 1}\left(t-\Delta_{(k)}\right)($ см. $(1.24))$, компонента $h_{*, 1}(t)$ удовлетворяет также и уравнению с запаздыванием

$$
\dot{h}=-\alpha h+A_{(k)}(t) h+\lambda B_{(k)}(t) h\left(t-\Delta_{(k)}\right) .
$$

Проведенные построения показывают, что уравнение (1.25) заведомо имеет единичный мультипликатор. Сделаем в этом уравнении замену $\exp (\alpha t) h \rightarrow h$. Тогда единичный мультипликатор перейдет в мультипликатор $\exp \left(m \alpha \Delta_{(k)} / k\right)$, а само уравнение (1.25) - в уравнение (1.17) при $\varkappa=\lambda \exp \left(\alpha \Delta_{(k)}\right)$. Таким образом, с необходимостью найдется номер $s$, для которого

$$
\left.\nu_{s}(\varkappa)\right|_{\varkappa=\lambda \exp \left(\alpha \Delta_{(k)}\right)}=\exp \left(\frac{m \alpha \Delta_{(k)}}{k}\right)=\nu .
$$

Отсюда и из очевидного равенства $\nu^{k}=\varkappa^{m}$ вытекают требуемые соотношения (1.19), (1.20).

В случае, когда мультипликатор $\nu$ кратный, рассуждения аналогичны. Действительно, пусть данному мультипликатору отвечает $p$ линейно независимых решений Ляпунова-Флоке. Тогда эти решения можно записать в матричной форме $\exp (\alpha t) H(t)$, где столбцами $(m n \times p)$-матрицы $H(t)$ являются линейно 
независимые $T_{(k)}$-периодические вектор-функции. Далее, в силу инвариантности системы (1.16) под действием замен (1.22) здесь вместо (1.23) будет выполняться равенство

$$
\Lambda H\left(t+\Delta_{(k)}\right)=H(t) D
$$

с некоторой невырожденной постоянной $(p \times p)$-матрицей $D$.

Свойство (1.26) позволяет свести проблему обоснования формул (1.19), (1.20) к рассмотрению предыдущего случая. Для того чтобы сделать это, фиксируем некоторое собственное значение $\lambda$ матрицы $D$, а через е обозначим отвечающий ему собственный вектор. Тогда, как нетрудно увидеть, для вектор-функции $h_{*}(t)=H(t) e$ справедливо соотношение (1.23). Последующие рассуждения совпадают с изложенными выше.

Итак, мы установили, что любой мультипликатор $\nu$ системы (1.16) может быть представлен в виде (1.19), где $\varkappa$ удовлетворяет одному из уравнений (1.20). Убедимся теперь в справедливости обратного утверждения. В связи с этим предположим, что уравнение (1.20) с номером $s=s_{0}$ допускает корень $\varkappa=\varkappa_{0} \neq 0$. Тогда уравнение

$$
\dot{h}=-\alpha h+A_{(k)}(t) h+\varkappa_{0} \exp \left(-\alpha \Delta_{(k)}\right) B_{(k)}(t) h\left(t-\Delta_{(k)}\right)
$$

при

$$
\alpha=\frac{k}{m \Delta_{(k)}}\left(\ln \left(\rho_{0}\right)+i \varphi_{0}\right), \quad \rho_{0}>0, \quad 0 \leqslant \varphi_{0}<2 \pi,
$$

где $\rho_{0} \exp \left(i \varphi_{0}\right)=\nu_{s_{0}}\left(\varkappa_{0}\right)$, имеет нетривиальное $T_{(k)}$-периодическое решение $\widetilde{h}(t)$. Далее, введем в рассмотрение величину

$$
\lambda=\varkappa_{0} \exp \left(-\alpha \Delta_{(k)}\right)
$$

и заметим, что в силу $(1.28)$ и соотношения $\varkappa_{0}^{m}=\left[\nu_{s_{0}}\left(\varkappa_{0}\right)\right]^{k}$ указанное значение параметра $\lambda$ удовлетворяет требуемому равенству $\lambda^{m}=1$ (см. (1.24)). Отсюда, в свою очередь, следует, что при выбранном $\lambda$ уравнения (1.27), (1.25) совпадают.

На заключительном этапе доказательства введем в рассмотрение функцию $h_{*, 1}(t)=\widetilde{h}(t)$, а остальные компоненты $h_{*, j}(t), j=2, \ldots, m$, определим посредством равенств (1.24), (1.29). Из установленной выше связи между уравнениями (1.27) и (1.25) вытекает, что в итоге получится решение Ляпунова-Флоке (1.21) исходной системы (1.16), отвечающее мультипликатору

$$
\nu=\exp \left(\frac{m \alpha \Delta_{(k)}}{k}\right)=\nu_{s_{0}}\left(\varkappa_{0}\right)
$$

Лемма доказана.

Леммы 1.1, 1.2 доставляют некую общую методику исследования периодических решений типа бегущих волн в кольцевых системах (1.10). Действительно, вопрос о существовании циклов вида (1.9) сводится к отысканию цикла $x_{*}(t, \Delta)$ вспомогательного уравнения с запаздыванием (1.11) и нахождению корней уравнений (1.12). Вопрос об устойчивости бегущих волн решается отдельно и в силу леммы 1.2 состоит в анализе расположения корней уравнений (1.20). Следует также отметить, что, хотя количество уравнений в системе (1.20), вообще говоря, счетно, совокупность всех их ненулевых корней 
заведомо конечна (в противном случае конечномерная система (1.16) имела бы счетное число различных мультипликаторов, что невозможно).

Ясно, что проблемы анализа вспомогательных уравнений (1.11), (1.17), лежащие в основе описанной выше методики, в общем случае нелокальны. Тем не менее, в некоторых ситуациях, когда возможно применить какие-либо асимптотические методы, с данными проблемами удается справиться. Точнее, это удается сделать в случае, когда для цикла вспомогательной системы (1.11) и его периода имеются хорошие приближенные формулы (с точностью до подходящих асимптотически малых добавок по некоторому параметру).

Одна из таких ситуаций состоит в следующем: в каждой отдельно взятой парциальной системе происходит бифуркация Андронова-Хопфа, а связь между системами слабая (упомянутый случай требует отдельного исследования, выходящего за рамки настоящей статьи). Другой крайний вариант, который, собственно, и реализуется в рассматриваемой ниже вспомогательной системе $(2.1),-$ возникновение цикла из бесконечности при $\mu=0$. Именно по этой причине в формулировках теорем о существовании и устойчивости бегущих волн мы исключаем из рассмотрения случай $\mu=0$.

С помощью изложенной выше общей методики далее устанавливается, что при увеличении $m$ и согласованном уменьшении параметров $\varepsilon, \mu$ в исходной системе (1.8) происходит неограниченное накапливание устойчивых бегущих волн, т. е. наблюдается феномен буферности.

\section{§ 2. Вспомогательные конструкции}

2.1. Анализ предельного релейного уравнения. Обратимся сначала к вспомогательному уравнению (1.11), которое в случае системы (1.8) имеет вид

$$
\ddot{x}+2 \mu \dot{x}+x=F\left(\frac{x(t-\Delta)}{\varepsilon}\right) .
$$

Наша ближайшая задача - показать, что при любом фиксированном значении запаздывания $\Delta$ из интервала

$$
0<\Delta<\pi
$$

и при независимых достаточно малых $\varepsilon, \mu>0$ это уравнение имеет нетривиальное (не равное нулю тождественно) периодическое решение.

Исследование уравнения (2.1) базируется на факте существования при $\varepsilon \rightarrow 0$ предельного объекта. Действительно, опираясь на свойства (1.6), (1.7) функции $F(x)$, замечаем, что

$$
\lim _{\varepsilon \rightarrow 0} F\left(\frac{x}{\varepsilon}\right)=-\operatorname{sign} x .
$$

Учитывая затем соотношение (2.3) в (2.1), убеждаемся в том, что при $\varepsilon \rightarrow 0$ уравнение (2.1) переходит в релейное уравнение с запаздыванием

$$
\ddot{x}+2 \mu \dot{x}+x=-\operatorname{sign} x(t-\Delta) .
$$


Как и в работах [10]-[12], понятие решения уравнения (2.4) определим конструктивно. С этой целью зададим множество $\Omega \subset C[-\Delta, 0] \times \mathbb{R}$ посредством равенства

$$
\Omega=\{(\varphi(t), z) \in C[-\Delta, 0] \times \mathbb{R}: \varphi(t)<0 \forall t \in[-\Delta, 0), \varphi(0)=0, z>0\}
$$

и для произвольно фиксированного элемента $(\varphi, z)$ из этого множества обозначим через $x=x_{\varphi, z}(t, \mu), t \geqslant 0$, решение уравнения $(2.4)$ с начальными условиями

$$
x=\frac{\varphi(t)}{\mu}, \quad-\Delta \leqslant t \leqslant 0,\left.\quad \dot{x}\right|_{t=0}=\frac{z}{\mu} .
$$

Заметим, что при $t \in[0, \Delta)$ в силу $(2.5),(2.6)$ выполняется неравенство $x(t-\Delta)<0$. Поэтому на промежутке времени $[0, \Delta)$ решение $x_{\varphi, z}(t, \mu)$ совпадает с решением задачи Коши

$$
\ddot{x}+2 \mu \dot{x}+x=1,\left.\quad x\right|_{t=0}=0,\left.\quad \dot{x}\right|_{t=0}=\frac{z}{\mu},
$$

а значит, имеет вид

$$
x_{\varphi, z}(t, \mu)=\frac{\theta_{1}(t, z, \mu)}{\mu},
$$

где $\theta_{1}(t, z, \mu)$ - решение задачи Коши

$$
\ddot{\theta}+2 \mu \dot{\theta}+\theta=\mu,\left.\quad \theta\right|_{t=0}=0,\left.\quad \dot{\theta}\right|_{t=0}=z .
$$

Отметим, кроме того, что при любом фиксированном $z>0$ фигурирующая в соотношении $(2.8)$ функция $\theta_{1}(t, z, \mu)$ раскладывается в сходящийся при всех $\mu \geqslant 0, t \geqslant 0$ ряд

$$
\theta_{1}(t, z, \mu)=\theta_{1,0}(t, z)+\mu \theta_{1,1}(t, z)+\mu^{2} \theta_{1,2}(t, z)+\cdots,
$$

где

$$
\begin{gathered}
\theta_{1,0}=z \sin t, \quad \theta_{1,1}=1-\cos t-z t \sin t, \\
\theta_{1,2}=\frac{1}{2}(z-2)(\sin t-t \cos t)+\frac{z}{2} t^{2} \sin t, \quad \ldots .
\end{gathered}
$$

Сходимость ряда (2.10) вытекает из факта аналитичности по $\mu$ задачи Коши (2.9). Точнее, выписывая явно решение этой задачи, нетрудно увидеть, что оно является аналитической функцией переменных $(t, \mu) \in \mathbb{C}^{2}$. Факт сходимости указанного ряда влечет за собой и сходимость последующих рядов (2.13), (2.16), (2.19), (2.25).

При $t=\Delta$ первый раз происходит переключение, и при $t \geqslant \Delta$ решение $x_{\varphi, z}(t, \mu)$ определяется уже из задачи Коши

$$
\ddot{x}+2 \mu \dot{x}+x=-1,\left.\quad x\right|_{t=\Delta}=\frac{\theta_{1}(\Delta, z, \mu)}{\mu},\left.\quad \dot{x}\right|_{t=\Delta}=\frac{\dot{\theta}_{1}(\Delta, z, \mu)}{\mu} .
$$

В свою очередь, решение этой задачи может быть представлено в аналогичном соотношению (2.8) виде

$$
x_{\varphi, z}(t, \mu)=\frac{\theta_{2}(t, z, \mu)}{\mu},
$$


где $\theta_{2}(t, z, \mu)$ - решение аналогичной (2.9) задачи Коши

$$
\ddot{\theta}+2 \mu \dot{\theta}+\theta=-\mu,\left.\quad \theta\right|_{t=\Delta}=\theta_{1}(\Delta, z, \mu),\left.\quad \dot{\theta}\right|_{t=\Delta}=\dot{\theta}_{1}(\Delta, z, \mu) .
$$

Опираясь, далее, на соотношения (2.10), (2.11), приходим к выводу, что функция $\theta_{2}(t, z, \mu)$ из $(2.12)$ разлагается в аналогичный $(2.10)$ сходящийся при всех $\mu \geqslant 0, t \geqslant \Delta$ ряд

$$
\theta_{2}(t, z, \mu)=\theta_{2,0}(t, z)+\mu \theta_{2,1}(t, z)+\mu^{2} \theta_{2,2}(t, z)+\cdots,
$$

где

$$
\begin{gathered}
\theta_{2,0}=z \sin t, \quad \theta_{2,1}=2 \cos (t-\Delta)-\cos t-1-z t \sin t \\
\theta_{2,2}=\theta_{1,2}-2(t-\Delta) \cos (t-\Delta)+2 \sin (t-\Delta) .
\end{gathered}
$$

Следует, однако, напомнить, что формула (2.12) выведена нами при априорном условии

$$
x_{\varphi, z}(t-\Delta, \mu)>0 .
$$

Нетрудно увидеть, что это условие заведомо справедливо на полуинтервале $\Delta<t \leqslant 2 \Delta$, поскольку в силу $(2.8),(2.10),(2.11)$ и ограничения (2.2) для параметра $\Delta$ имеем $x_{\varphi, z}(t, \mu)>0$ при $t \in(0, \Delta]$. Ясно, что неравенство (2.15), а значит, и представление (2.12) сохраняются до очередного момента переключения $t=t_{0}+\Delta$, где $t_{0}=t_{0}(z, \mu)$ - наименьший корень уравнения $\theta_{2}(t, z, \mu)=0$ из промежутка $[\Delta, \infty)$. Объединяя соотношения (2.13), (2.14) с условием $\Delta<\pi$ (см. (2.2)) и опираясь на теорему о неявной функции (в аналитическом ее варианте), убеждаемся в том, что этот корень разлагается в сходящийся при всех достаточно малых $\mu \geqslant 0$ ряд

$$
t_{0}(z, \mu)=\pi+t_{0,1}(z) \mu+t_{0,2}(z) \mu^{2}+\cdots, \quad t_{0,1}=-\frac{2 \cos \Delta}{z} .
$$

Для построения решения $x_{\varphi, z}(t, \mu)$ при $t \geqslant t_{0}+\Delta$ воспользуемся симметрией уравнения (2.4), а именно тем фактом, что оно не меняется при замене

$$
(x, \dot{x}) \rightarrow-(x, \dot{x}) .
$$

С этой целью введем в рассмотрение одномерное отображение

$$
z \rightarrow \Phi_{\mu}(z) \stackrel{\text { def }}{=}-\left.\dot{\theta}_{2}(t, z, \mu)\right|_{t=t_{0}(z, \mu)},
$$

которое в силу $(2.13),(2.14),(2.16)$ допускает представление в виде сходящегося при малых $\mu$ ряда

$$
\Phi_{\mu}(z)=z+\mu \Phi_{1}(z)+\mu^{2} \Phi_{2}(z)+\cdots
$$

где

$$
\Phi_{1}=2 \sin \Delta-\pi z, \quad \Phi_{2}=\frac{2 \cos \Delta}{z}(\cos \Delta+2 z-1)+\frac{\pi^{2}}{2} z-2(\pi-\Delta) \sin \Delta .
$$

Выполним в уравнении (2.4) указанную выше замену (2.17) и сдвиг по времени $t-t_{0} \rightarrow t$. В результате приходим к аналогичной $(2.4),(2.6)$ задаче Коши

$$
\begin{gathered}
\ddot{x}+2 \mu \dot{x}+x=-\operatorname{sign} x(t-\Delta), \\
x=-x_{\varphi, z}\left(t+t_{0}(z, \mu), \mu\right), \quad-\Delta \leqslant t \leqslant 0,\left.\quad \dot{x}\right|_{t=0}=\frac{\bar{z}}{\mu},
\end{gathered}
$$


где $\bar{z}=\Phi_{\mu}(z)$. Заметим, далее, что из проделанных выше построений вытекает справедливость включения

$$
\left(-\mu x_{\varphi, z}\left(t+t_{0}(z, \mu), \mu\right), \bar{z}\right) \in \Omega
$$

где $\Omega$ - множество (2.5). Поэтому для решения задачи $(2.21)$ на отрезках времени $t \in[0, \Delta]$ и $t \in\left[\Delta, t_{0}(\bar{z}, \mu)+\Delta\right]$ имеют место формулы $(2.8)$ и (2.12) соответственно, в которых $z$ необходимо заменить на $\bar{z}$. Отсюда, в свою очередь, следует, что решение $x_{\varphi, z}(t, \mu)$ исходной задачи Коши $(2.4),(2.6)$ допускает представления

$$
\begin{aligned}
& x_{\varphi, z}(t, \mu)= \\
& \quad=\left\{\begin{aligned}
-\frac{\theta_{1}\left(t-t_{0}(z, \mu), \bar{z}, \mu\right)}{\mu} & \text { при } t \in\left[t_{0}(z, \mu), t_{0}(z, \mu)+\Delta\right], \\
-\frac{\theta_{2}\left(t-t_{0}(z, \mu), \bar{z}, \mu\right)}{\mu} & \text { при } t \in\left[t_{0}(z, \mu)+\Delta, t_{0}(z, \mu)+t_{0}(\bar{z}, \mu)+\Delta\right] .
\end{aligned}\right.
\end{aligned}
$$

Итак, предпринятый анализ свидетельствует о том, что на множестве (2.5) определен оператор последования Пуанкаре

$$
\Pi_{\mu}:(\varphi(t), z) \rightarrow\left(\mu x_{\varphi, z}\left(t+t_{0}(z, \mu)+t_{0}(\bar{z}, \mu), \mu\right), \Phi_{\mu}(\bar{z})\right), \quad-\Delta \leqslant t \leqslant 0 .
$$

При этом, что представляет интерес, различные функции $\varphi(t)$ (при фиксированном значении $z$ ) переходят в одну и ту же функцию

$$
\mu x_{\varphi, z}\left(t+t_{0}(z, \mu)+t_{0}(\bar{z}, \mu), \mu\right), \quad-\Delta \leqslant t \leqslant 0,
$$

которая в силу (2.22) на самом деле не зависит от $\varphi(t)$. Таким образом, оператор (2.23) представляет собой надстройку над соответствующим одномерным отображением

$$
z \rightarrow \Phi_{\mu}^{2}(z)
$$

где $\Phi_{\mu}^{2}=\Phi_{\mu} \circ \Phi_{\mu}$.

Теперь получаем, что вопрос о неподвижных точках отображения $\Pi_{\mu}$ сводится к аналогичному вопросу для отображения (2.24). Далее, опираясь на соотношения $(2.19),(2.20)$, замечаем, что отображение (2.18) допускает экспоненциально устойчивую неподвижную точку $z=z_{*}(\mu)$, разлагающуюся в сходящийся при малых $\mu$ ряд

$$
\begin{aligned}
z_{*}(\mu) & =z_{0}+\mu z_{1}+\cdots, \\
z_{0}=\frac{2}{\pi} \sin \Delta>0, \quad z_{1} & =\frac{2}{\pi}(2 \cos \Delta+\Delta \sin \Delta)-\frac{\sin (3 \Delta / 2)}{\cos (\Delta / 2)} .
\end{aligned}
$$

Поскольку точка (2.25) является неподвижной и для отображения (2.24), то в исходном релейном уравнении (2.4) ей отвечает экспоненциально орбитально устойчивый цикл $x=x_{*}(t, \mu)$ периода $T_{*}(\mu)$. При этом функции $T_{*}(\mu)$ и $x_{*}(t, \mu)$ 
задаются равенствами

$$
\begin{gathered}
T_{*}(\mu)=2 t_{*}(\mu), \quad t_{*}(\mu)=\left.t_{0}(z, \mu)\right|_{z=z_{*}(\mu)}=\pi-\pi \operatorname{ctg}(\Delta) \mu+O\left(\mu^{2}\right), \\
x_{*}(t, \mu)= \begin{cases}\frac{\theta_{1, *}(t, \mu)}{\mu} & \text { при } 0 \leqslant t \leqslant \Delta, \\
\frac{\theta_{2, *}(t, \mu)}{\mu} & \text { при } \Delta \leqslant t \leqslant t_{*}(\mu), \\
& x_{*}\left(t+t_{*}(\mu), \mu\right) \equiv-x_{*}(t, \mu),\end{cases}
\end{gathered}
$$

где $\theta_{j, *}(t, \mu)=\left.\theta_{j}(t, z, \mu)\right|_{z=z_{*}(\mu)}, j=1,2$.

Перейдем к изучению вопроса о связи между периодическими решениями уравнений (2.1) и (2.4). Справедливо следующее утверждение.

Теорема 2.1. Предположим, что параметр $\Delta$ фиксирован и удовлетворяет условию (2.2). Тогда найдутся такие достаточно малые положительные $\varepsilon_{0}=\varepsilon_{0}(\Delta), \mu_{0}=\mu_{0}(\Delta)$, что при всех $0<\varepsilon \leqslant \varepsilon_{0}, 0<\mu \leqslant \mu_{0}$ уравнение (2.1) имеет нетривиальное периодическое решение $x=x(t, \varepsilon, \mu)$, $x(0, \varepsilon, \mu) \equiv 0$, периода $T(\varepsilon, \mu)$. Для этого решения при $\varepsilon, \mu \rightarrow 0$ справедливь асимптотические представления

$$
\begin{gathered}
\max _{0 \leqslant t \leqslant T(\varepsilon, \mu)}\left\{\mu\left|x(t, \varepsilon, \mu)-x_{*}(t, \mu)\right|+\mu\left|\dot{x}(t, \varepsilon, \mu)-\dot{x}_{*}(t, \mu)\right|\right\}=O\left(\varepsilon \mu \ln \frac{1}{\varepsilon \mu}\right), \\
T(\varepsilon, \mu)=T_{*}(\mu)+O\left(\varepsilon \mu^{2} \ln \frac{1}{\varepsilon \mu}\right),
\end{gathered}
$$

где $x_{*}(t, \mu), T_{*}(\mu)-$ функиии (2.26).

Доказательство теоремы 2.1, приводящееся ниже, опирается на некоторые дополнительные конструкции. Для их описания фиксируем произвольно постоянные $q_{1}, q_{2}, 0<q_{1}<q_{2}$, и $z_{1}, z_{2}, 0<z_{1}<z_{2}$, и обозначим через $\Omega\left(q_{1}, q_{2}, z_{1}, z_{2}\right) \subset C[-\Delta, 0] \times \mathbb{R}$ замкнутое, ограниченное и выпуклое множество пар $(\varphi(t), z)$, удовлетворяющих требованиям

$$
q_{2} t \leqslant \varphi(t) \leqslant q_{1} t \quad \forall t \in[-\Delta, 0], \quad z_{1} \leqslant z \leqslant z_{2} .
$$

Далее, для произвольного элемента $(\varphi(t), z) \in \Omega\left(q_{1}, q_{2}, z_{1}, z_{2}\right)$ рассмотрим решение $x=x_{\varphi, z}(t, \varepsilon, \mu), t \geqslant 0$, уравнения (2.1) с начальными условиями

$$
x=\frac{\varphi(t)}{\mu}, \quad-\Delta \leqslant t \leqslant 0,\left.\quad \dot{x}\right|_{t=0}=\frac{z}{\mu},
$$

а через $t_{\varphi, z}^{(1)}(\varepsilon, \mu)$ и $t_{\varphi, z}^{(2)}(\varepsilon, \mu)$ обозначим первый и второй положительные корни уравнения

$$
x_{\varphi, z}(t, \varepsilon, \mu)=0
$$

соответственно (если они существуют). И наконец, зададим аналогичный оператору (2.23) оператор последования $\Pi_{\varepsilon, \mu}: \Omega\left(q_{1}, q_{2}, z_{1}, z_{2}\right) \rightarrow C[-\Delta, 0] \times \mathbb{R}$ с помощью равенства

$$
\begin{aligned}
& \Pi_{\varepsilon, \mu}[(\varphi, z)]= \\
& \quad=\left(\mu x_{\varphi, z}\left(t+t_{\varphi, z}^{(2)}(\varepsilon, \mu), \varepsilon, \mu\right), \mu \dot{x}_{\varphi, z}\left(t_{\varphi, z}^{(2)}(\varepsilon, \mu), \varepsilon, \mu\right)\right), \quad-\Delta \leqslant t \leqslant 0 .
\end{aligned}
$$


Последующий план действий таков. Сначала мы установим равномерную по $(\varphi, z) \in \Omega\left(q_{1}, q_{2}, z_{1}, z_{2}\right)$ асимптотическую близость решения $x_{\varphi, z}(t, \varepsilon, \mu)$ к решению $x_{\varphi, z}(t, \mu)$ релейного уравнения (2.4) с теми же начальными условиями $(\varphi, z)$ на различных промежутках изменения $t$. Затем покажем, что при подходящем выборе параметров $q_{j}, z_{j}, j=1,2$, оператор (2.31) преобразует множество $\Omega\left(q_{1}, q_{2}, z_{1}, z_{2}\right)$ в себя. В заключение убедимся, что интересующее нас периодическое решение $x(t, \varepsilon, \mu)$ (существование которого вытекает из принципа Шаудера) обладает требуемыми асимптотическими свойствами (2.27).

2.2. Доказательство теоремы 2.1. Для построения асимптотики функции $x_{\varphi, z}(t, \varepsilon, \mu)$ рассмотрим сначала отрезок времени $0 \leqslant t \leqslant \Delta$. Согласно уравнениям $(2.1),(2.29)$ на этом отрезке $x_{\varphi, z}(t, \varepsilon, \mu)$ является решением задачи Коши

$$
\ddot{x}+2 \mu \dot{x}+x=F\left(\frac{\varphi(t-\Delta)}{\varepsilon \mu}\right),\left.\quad x\right|_{t=0}=0,\left.\quad \dot{x}\right|_{t=0}=\frac{z}{\mu} .
$$

Тем самым имеет место формула

$$
x_{\varphi, z}(t, \varepsilon, \mu)=x_{\varphi, z}(t, \mu)+\int_{0}^{t} K(t-\tau, \mu)\left[F\left(\frac{\varphi(\tau-\Delta)}{\varepsilon \mu}\right)-1\right] d \tau,
$$

где $K(t, \mu)=\omega^{-1} \exp (-\mu t) \sin (\omega t), \omega=\sqrt{1-\mu^{2}}$, а $x_{\varphi, z}(t, \mu)$ - решение задачи Коши $(2.4),(2.6)$. Далее, из свойств (1.7) функции $F(x)$ выводим неравенство

$$
|F(x)-1| \leqslant \frac{M}{1+|x|} \quad \forall x \leqslant 0 ;
$$

здесь и далее через $M, M_{1}, M_{2}, \ldots$ обозначены различные универсальные положительные постоянные, точные значения которых несущественны. В свою очередь, неравенство (2.33) вместе с оценками для $\varphi(t)$ из $(2.28)$ позволяет заключить, что

$$
\left|F\left(\frac{\varphi(t-\Delta)}{\varepsilon \mu}\right)-1\right| \leqslant \frac{M}{1+|t-\Delta| /(\varepsilon \mu)} \quad \forall t \in[0, \Delta] .
$$

Отсюда и из (2.32) вытекает, что при $0 \leqslant t \leqslant \Delta$ выполняются следующие асимптотические представления:

$$
\begin{gathered}
x_{\varphi, z}(t, \varepsilon, \mu)=x_{\varphi, z}(t, \mu)+O\left(\varepsilon \mu \ln \frac{1}{\varepsilon \mu}\right), \\
\dot{x}_{\varphi, z}(t, \varepsilon, \mu)=\dot{x}_{\varphi, z}(t, \mu)+O\left(\varepsilon \mu \ln \frac{1}{\varepsilon \mu}\right), \quad \varepsilon, \mu \rightarrow 0 .
\end{gathered}
$$

Добавим, что здесь и во всех других случаях, когда оказываются справедливыми равенства (2.34), остатки в них равномерны по начальным условиям $(\varphi, z) \in \Omega\left(q_{1}, q_{2}, z_{1}, z_{2}\right)$ и по $t$ из соответствующего промежутка времени.

При $\Delta \leqslant t \leqslant 2 \Delta$ рассмотрению подлежит задача Коши

$$
\begin{gathered}
\ddot{x}+2 \mu \dot{x}+x=F\left(\frac{x_{\varphi, z}(t-\Delta, \varepsilon, \mu)}{\varepsilon}\right), \\
\left.x\right|_{t=\Delta}=x_{\varphi, z}(\Delta, \varepsilon, \mu),\left.\quad \dot{x}\right|_{t=\Delta}=\dot{x}_{\varphi, z}(\Delta, \varepsilon, \mu),
\end{gathered}
$$


неоднородность в которой и начальные условия уже известны из предыдущего шага. Поэтому решение $x_{\varphi, z}(t, \varepsilon, \mu)$ этой задачи мы можем представить в аналогичном (2.32) виде

$$
\begin{array}{r}
x_{\varphi, z}(t, \varepsilon, \mu)=x_{\varphi, z}(t, \mu)+\int_{\Delta}^{t} K(t-\tau, \mu)\left[F\left(\frac{x_{\varphi, z}(\tau-\Delta, \varepsilon, \mu)}{\varepsilon}\right)+1\right] d \tau \\
+\exp (-\mu(t-\Delta))\left[c_{1} \cos (\omega(t-\Delta))+c_{2} \sin (\omega(t-\Delta))\right],
\end{array}
$$

где

$$
\begin{gathered}
c_{1}=\left.\left(x_{\varphi, z}(t, \varepsilon, \mu)-x_{\varphi, z}(t, \mu)\right)\right|_{t=\Delta}, \\
\omega c_{2}-\mu c_{1}=\left.\left(\dot{x}_{\varphi, z}(t, \varepsilon, \mu)-\dot{x}_{\varphi, z}(t, \mu)\right)\right|_{t=\Delta} .
\end{gathered}
$$

Отметим, что в силу уже установленных ранее асимптотических соотношений (2.34) при $0 \leqslant t \leqslant \Delta$ и формул (2.36) третье слагаемое из (2.35) и его производная по $t$ имеют порядок $\varepsilon \mu \ln (1 /(\varepsilon \mu))$. Далее, для исследования фигурирующего в (2.35) интегрального слагаемого воспользуемся вытекающим из (1.7) неравенством

$$
|F(x)+1| \leqslant \frac{M}{1+x} \quad \forall x \geqslant 0 .
$$

Объединяя (2.37) с оценкой вида

$$
x_{\varphi, z}(t-\Delta, \varepsilon, \mu) \geqslant \frac{M(t-\Delta)}{\mu} \quad \forall t \in[\Delta, 2 \Delta],
$$

справедливой в силу (2.34) и известных свойств решения $x_{\varphi, z}(t, \mu)$ релейного уравнения (2.4) (см. (2.8), (2.10), (2.11)), приходим к выводу, что

$$
\left|F\left(\frac{x_{\varphi, z}(t-\Delta, \varepsilon, \mu)}{\varepsilon}\right)+1\right| \leqslant \frac{M}{1+(t-\Delta) /(\varepsilon \mu)} \quad \forall t \in[\Delta, 2 \Delta] .
$$

Отсюда очевидным образом следует, что упомянутое выше интегральное слагаемое имеет порядок $\varepsilon \mu \ln (1 /(\varepsilon \mu))$ в метрике $C^{1}[\Delta, 2 \Delta]$.

Итак, мы убедились в том, что асимптотические представления (2.34) сохраняются на отрезке времени $\Delta \leqslant t \leqslant 2 \Delta$. Однако описанная выше методика позволяет распространить их и далее, а именно на отрезок $2 \Delta \leqslant t \leqslant \Delta+\pi-\delta$, где $\delta \in(0, \min (\Delta, \pi-\Delta))$ произвольно фиксировано. Сделать это удается с помощью так называемого метода шагов, суть которого состоит в следующем. Разобьем указанный отрезок на части длины не более $\Delta$ и на очередном промежутке, например, при $2 \Delta \leqslant t \leqslant 3 \Delta$, опираясь на равенства (2.34) (уже установленные на предыдущем участке изменения $t$ ), сначала получаем оценку вида $x_{\varphi, z}(t-\Delta, \varepsilon, \mu) \geqslant M / \mu, M=$ const $>0$, а затем, выписывая аналогичные $(2.35),(2.36)$ формулы для нового момента времени (например, для $t=2 \Delta$ ) и анализируя их подобно тому, как это было сделано выше, распространяем асимптотические представления (2.34) на шаг вперед. Ясно, что описанный процесс продолжается до тех пор, пока $x_{\varphi, z}(t-\Delta, \mu) \geqslant M / \mu, M=$ const $>0$. Тем самым, рассуждая подобным образом, мы заведомо добираемся до точки $t=\Delta+\pi-\delta$.

Теперь найдем интересующий нас первый положительный корень $t=$ $t_{\varphi, z}^{(1)}(\varepsilon, \mu)$ уравнения (2.30). Соотношения (2.34) наводят на мысль, что он должен быть близок к аналогичному корню $t=t_{0}(z, \mu)$ (см. (2.16)) уравнения 
$x_{\varphi, z}(t, \mu)=0$. Это действительно так, поскольку в силу выбора $\delta$ точка $t=\pi$ принадлежит отрезку времени $[\Delta, \Delta+\pi-\delta]$, на котором формулы (2.34) уже установлены. Дополняя их представлениями $(2.12)-(2.14)$, для $t_{\varphi, z}^{(1)}(\varepsilon, \mu)$ получаем равномерное по $(\varphi, z) \in \Omega\left(q_{1}, q_{2}, z_{1}, z_{2}\right)$ асимптотическое равенство

$$
t_{\varphi, z}^{(1)}(\varepsilon, \mu)=t_{0}(z, \mu)+O\left(\varepsilon \mu^{2} \ln \frac{1}{\varepsilon \mu}\right), \quad \varepsilon, \mu \rightarrow 0 .
$$

На очередных двух отрезках

$$
t_{\varphi, z}^{(1)} \leqslant t \leqslant t_{\varphi, z}^{(1)}+\Delta, \quad t_{\varphi, z}^{(1)}+\Delta \leqslant t \leqslant t_{\varphi, z}^{(1)}+2 \Delta
$$

рассуждения однотипны. Действительно, отталкиваясь от уже известных асимптотических представлений для $x_{\varphi, z}(t, \varepsilon, \mu), x_{\varphi, z}(t, \mu)$ при $0 \leqslant t \leqslant t_{\varphi, z}^{(1)}$ (см. (2.34), (2.8), (2.10)-(2.14)), последовательно убеждаемся в том, что

$$
\begin{gathered}
x_{\varphi, z}(t-\Delta, \varepsilon, \mu) \geqslant \frac{M\left(t_{\varphi, z}^{(1)}+\Delta-t\right)}{\mu}, \\
\left|F\left(\frac{x_{\varphi, z}(t-\Delta, \varepsilon, \mu)}{\varepsilon}\right)+1\right| \leqslant \frac{M}{1+\left(t_{\varphi, z}^{(1)}+\Delta-t\right) /(\varepsilon \mu)} \quad \forall t \in\left[t_{\varphi, z}^{(1)}, t_{\varphi, z}^{(1)}+\Delta\right] .
\end{gathered}
$$

Учтем теперь вторую из этих оценок в равенствах, получающихся из формул $(2.35),(2.36)$ при замене начального момента времени $t=\Delta$ на $t=t_{\varphi, z}^{(1)}$. В результате приходим к требуемым асимптотическим соотношениям (2.34) на новом отрезке $t_{\varphi, z}^{(1)} \leqslant t \leqslant t_{\varphi, z}^{(1)}+\Delta$.

Случай $t_{\varphi, z}^{(1)}+\Delta \leqslant t \leqslant t_{\varphi, z}^{(1)}+2 \Delta$ разбирается аналогично. Здесь справедливость равенств (2.34) устанавливается посредством оценок

$$
\begin{gathered}
x_{\varphi, z}(t-\Delta, \varepsilon, \mu) \leqslant-\frac{M\left(t-\Delta-t_{\varphi, z}^{(1)}\right)}{\mu} \\
\left|F\left(\frac{x_{\varphi, z}(t-\Delta, \varepsilon, \mu)}{\varepsilon}\right)-1\right| \leqslant \frac{M}{1+\left(t-t_{\varphi, z}^{(1)}-\Delta\right) /(\varepsilon \mu)} \quad \forall t \in\left[t_{\varphi, z}^{(1)}+\Delta, t_{\varphi, z}^{(1)}+2 \Delta\right]
\end{gathered}
$$

и формул

$$
\begin{aligned}
& x_{\varphi, z}(t, \varepsilon, \mu)=x_{\varphi, z}(t, \mu)+\int_{t_{\varphi, z}^{(1)}+\Delta}^{t} K(t-\tau, \mu)\left[F\left(\frac{x_{\varphi, z}(\tau-\Delta, \varepsilon, \mu)}{\varepsilon}\right)-1\right] d \tau \\
& \quad+\exp \left(-\mu\left(t-t_{\varphi, z}^{(1)}-\Delta\right)\right)\left[c_{1} \cos \left(\omega\left(t-t_{\varphi, z}^{(1)}-\Delta\right)\right)+c_{2} \sin \left(\omega\left(t-t_{\varphi, z}^{(1)}-\Delta\right)\right)\right],
\end{aligned}
$$

где

$$
\begin{gathered}
c_{1}=\left.\left(x_{\varphi, z}(t, \varepsilon, \mu)-x_{\varphi, z}(t, \mu)\right)\right|_{t=t_{\varphi, z}^{(1)}+\Delta}, \\
\omega c_{2}-\mu c_{1}=\left.\left(\dot{x}_{\varphi, z}(t, \varepsilon, \mu)-\dot{x}_{\varphi, z}(t, \mu)\right)\right|_{t=t_{\varphi, z}^{(1)}+\Delta}
\end{gathered}
$$

На завершающем этапе построения асимптотики решения $x_{\varphi, z}(t, \varepsilon, \mu)$ обратимся к отрезку $t_{\varphi, z}^{(1)}+2 \Delta \leqslant t \leqslant \Delta+2 \pi-\delta$, где $\delta=$ const $\in(0, \min (\Delta, \pi-\Delta))$. 
Как и в случае отрезка $[2 \Delta, \Delta+\pi-\delta]$, рассуждения здесь базируются на методе шагов. А именно, при рассмотрении очередного промежутка времени длины $\Delta$, опираясь на формулы (2.34), установленные на предыдущем шаге, сначала получаем оценку вида

$$
x_{\varphi, z}(t-\Delta, \varepsilon, \mu) \leqslant-\frac{M}{\mu},
$$

а затем, привлекая аналогичные (2.39), (2.40) равенства и вытекающую из соотношений $(2.41),(2.33)$ оценку

$$
\left|F\left(\frac{x_{\varphi, z}(t-\Delta, \varepsilon, \mu)}{\varepsilon}\right)-1\right| \leqslant M \varepsilon \mu,
$$

убеждаемся в справедливости требуемых соотношений (2.34) на текущем шаге изменения $t$. Отметим, что поскольку в силу (2.22) при всех $t$ из отрезка $\left[t_{\varphi, z}^{(1)}+2 \Delta, \Delta+2 \pi-\delta\right]$ выполняется неравенство

$$
x_{\varphi, z}(t-\Delta, \mu) \leqslant-\frac{M}{\mu}, \quad M=\text { const }>0,
$$

то, действуя указанным образом, мы заведомо доберемся до точки $t=\Delta+2 \pi-\delta$.

Как и выше, отдельного рассмотрения требует вопрос о существовании второго положительного корня $t=t_{\varphi, z}^{(2)}(\varepsilon, \mu)$ уравнения $(2.30)$. Из общих соображений ясно, что он должен быть близок к $2 \pi$. Действительно, так как точка $t=2 \pi$ принадлежит отрезку $\left[t_{\varphi, z}^{(1)}, \Delta+2 \pi-\delta\right]$, мы вправе воспользоваться уже установленными на этом промежутке времени асимптотическими равенствами $(2.34)$ и формулами $(2.22)$. В результате для $t_{\varphi, z}^{(2)}(\varepsilon, \mu)$ получаем равномерное по $(\varphi, z) \in \Omega\left(q_{1}, q_{2}, z_{1}, z_{2}\right)$ асимптотическое представление

$$
t_{\varphi, z}^{(2)}(\varepsilon, \mu)=t_{0}(z, \mu)+t_{0}(\bar{z}, \mu)+O\left(\varepsilon \mu^{2} \ln \frac{1}{\varepsilon \mu}\right), \quad \varepsilon, \mu \rightarrow 0,
$$

где, напомним, $\bar{z}=\Phi_{\mu}(z)$.

Подведем предварительный итог. Формулы (2.34), справедливые на отрезке времени $0 \leqslant t \leqslant \Delta+2 \pi-\delta$, и представление (2.42) свидетельствуют о том, что оператор (2.31) корректно определен на множестве $\Omega\left(q_{1}, q_{2}, z_{1}, z_{2}\right)$ и

$$
\begin{aligned}
& \sup _{(\varphi, z) \in \Omega\left(q_{1}, q_{2}, z_{1}, z_{2}\right)}\left\|\Pi_{\varepsilon, \mu}[(\varphi, z)]-\Pi_{\mu}[(\varphi, z)]\right\|_{C^{1}[-\Delta, 0] \times \mathbb{R}} \\
&=O\left(\varepsilon \mu^{2} \ln \frac{1}{\varepsilon \mu}\right), \quad \varepsilon, \mu \rightarrow 0 .
\end{aligned}
$$

Здесь $\Pi_{\mu}$ - оператор $(2.23)$, а норма в $C^{1}[-\Delta, 0] \times \mathbb{R}$ задана равенством

$$
\|(\varphi, z)\|_{C^{1}[-\Delta, 0] \times \mathbb{R}}=\max _{-\Delta \leqslant t \leqslant 0}|\varphi(t)|+\max _{-\Delta \leqslant t \leqslant 0}|\dot{\varphi}(t)|+|z| .
$$

На следующем этапе доказательства распорядимся имеющимися в запасе постоянными $q_{j}, z_{j}, j=1,2$. Будем считать, что $z_{1} \in\left(0, z_{0}\right), z_{2}>z_{0}$, где $z_{0}>0$ - константа из $(2.25)$, а $q_{1}, q_{2}>0$ подобраны таким образом, что

$$
q_{2}>z_{2}, \quad q_{1}<z_{1}, \quad q_{2} t<z \sin t<q_{1} t \quad \forall t \in[-\Delta, 0), \quad \forall z \in\left[z_{1}, z_{2}\right] .
$$


Убедимся, далее, в том, что при указанном выборе $q_{j}, z_{j}$ и при всех достаточно малых $\varepsilon, \mu>0$ оператор $\Pi_{\varepsilon, \mu}$ преобразует множество $\Omega\left(q_{1}, q_{2}, z_{1}, z_{2}\right)$ в себя.

Покажем сначала, что $\Pi_{\mu}\left(\Omega\left(q_{1}, q_{2}, z_{1}, z_{2}\right)\right) \subset \Omega\left(q_{1}, q_{2}, z_{1}, z_{2}\right)$. С этой целью обратимся к вытекающему из (2.19), (2.20) асимптотическому представлению

$$
\Phi_{\mu}^{2}(z)=z+2 \mu(2 \sin \Delta-\pi z)+O\left(\mu^{2}\right)
$$

равномерному по $z$ из любого конечного отрезка. Объединяя его с условиями $2 \sin \Delta-\pi z_{1}>0,2 \sin \Delta-\pi z_{2}<0$, наложенными на $z_{1}, z_{2}$, приходим к выводу, что при всех $z \in\left[z_{1}, z_{2}\right]$

$$
\begin{aligned}
& \Phi_{\mu}^{2}(z) \leqslant \Phi_{\mu}^{2}\left(z_{2}\right)=z_{2}+2 \mu\left(2 \sin \Delta-\pi z_{2}\right)+O\left(\mu^{2}\right)<z_{2}, \\
& \Phi_{\mu}^{2}(z) \geqslant \Phi_{\mu}^{2}\left(z_{1}\right)=z_{1}+2 \mu\left(2 \sin \Delta-\pi z_{1}\right)+O\left(\mu^{2}\right)>z_{1} .
\end{aligned}
$$

Обратимся теперь к первой компоненте оператора (2.23). Привлекая известные асимптотические свойства решения $x_{\varphi, z}(t, \mu)$ (см. п. 2.1), нетрудно увидеть, что для нее в метрике пространства $C^{1}[-\Delta, 0]$ выполняется асимптотическое равенство

$$
\mu x_{\varphi, z}\left(t+t_{0}(z, \mu)+t_{0}(\bar{z}, \mu), \mu\right)=z \sin t+O(\mu), \quad-\Delta \leqslant t \leqslant 0,
$$

равномерное по $z \in\left[z_{1}, z_{2}\right]$ и $\varphi$ (напомним, что функция $x_{\varphi, z}(t, \mu)$ на самом деле не зависит от $\varphi)$. Отсюда и из $(2.44)$ очевидным образом имеем

$$
q_{2} t<\mu x_{\varphi, z}\left(t+t_{0}(z, \mu)+t_{0}(\bar{z}, \mu), \mu\right)<q_{1} t \quad \forall t \in[-\Delta, 0), \quad \forall z \in\left[z_{1}, z_{2}\right] .
$$

Проделанные построения приводят к выводу, что оператор $\Pi_{\mu}$ действительно переводит множество $\Omega\left(q_{1}, q_{2}, z_{1}, z_{2}\right)$ в себя. В случае $\Pi_{\varepsilon, \mu}$ ситуация аналогичная. В самом деле, из (2.43), (2.45) следует, что

$$
\begin{aligned}
\mu \dot{x}_{\varphi, z}\left(t_{\varphi, z}^{(2)}(\varepsilon, \mu), \varepsilon, \mu\right) & =\Phi_{\mu}^{2}(z)+O\left(\varepsilon \mu^{2} \ln \frac{1}{\varepsilon \mu}\right) \\
& =z+2 \mu(2 \sin \Delta-\pi z)+O\left(\mu^{2}+\varepsilon \mu^{2} \ln \frac{1}{\varepsilon \mu}\right)
\end{aligned}
$$

а значит, выполняются аналогичные (2.46) оценки

$$
z_{1}<\mu \dot{x}_{\varphi, z}\left(t_{\varphi, z}^{(2)}(\varepsilon, \mu), \varepsilon, \mu\right)<z_{2} \quad \forall(\varphi, z) \in \Omega\left(q_{1}, q_{2}, z_{1}, z_{2}\right) .
$$

Сохраняется здесь и аналогичное (2.47) асимптотическое представление

$$
\mu x_{\varphi, z}\left(t+t_{\varphi, z}^{(2)}(\varepsilon, \mu), \varepsilon, \mu\right)=z \sin t+O(\mu), \quad-\Delta \leqslant t \leqslant 0,
$$

которое согласно (2.43) справедливо в метрике $C^{1}[-\Delta, 0]$ равномерно по начальным условиям $(\varphi, z) \in \Omega\left(q_{1}, q_{2}, z_{1}, z_{2}\right)$. Тем самым, остаются в силе аналогичные $(2.48)$ итоговые неравенства

$$
\begin{gathered}
q_{2} t<\mu x_{\varphi, z}\left(t+t_{\varphi, z}^{(2)}(\varepsilon, \mu), \varepsilon, \mu\right)<q_{1} t \quad \forall t \in[-\Delta, 0), \\
\forall(\varphi, z) \in \Omega\left(q_{1}, q_{2}, z_{1}, z_{2}\right) .
\end{gathered}
$$


Полученные выше соотношения (2.50), (2.51), собственно говоря, и означают, что справедливо требуемое свойство $\Pi_{\varepsilon, \mu}\left(\Omega\left(q_{1}, q_{2}, z_{1}, z_{2}\right)\right) \subset \Omega\left(q_{1}, q_{2}, z_{1}, z_{2}\right)$. Учитывая это обстоятельство и опираясь на принцип Шаудера, заключаем, что оператор $\Pi_{\varepsilon, \mu}$, очевидным образом являющийся компактным, имеет в множестве $\Omega\left(q_{1}, q_{2}, z_{1}, z_{2}\right)$ по крайней мере одну неподвижную точку

$$
(\widehat{\varphi}, \widehat{z})=(\widehat{\varphi}(t, \varepsilon, \mu), \widehat{z}(\varepsilon, \mu)) .
$$

Ясно также, что решение $x=x(t, \varepsilon, \mu)$ уравнения (2.1) с начальными условиями

$$
x=\frac{\widehat{\varphi}(t, \varepsilon, \mu)}{\mu}, \quad-\Delta \leqslant t \leqslant 0,\left.\quad \dot{x}\right|_{t=0}=\frac{\widehat{z}(\varepsilon, \mu)}{\mu}
$$

оказывается периодическим с периодом $T(\varepsilon, \mu)=\left.t_{\varphi, z}^{(2)}(\varepsilon, \mu)\right|_{(\varphi, z)=(\widehat{\varphi}, \widehat{z})}$.

Завершая доказательство теоремы 2.1, установим требуемые асимптотические равенства (2.27). В связи с этим обратим внимание на то, что в силу формулы (2.49) компонента $\widehat{z}(\varepsilon, \mu)$ найденной выше неподвижной точки удовлетворяет уравнению

$$
z=\Phi_{\mu}^{2}(z)+O\left(\varepsilon \mu^{2} \ln \frac{1}{\varepsilon \mu}\right),
$$

а значит, допускает асимптотику

$$
\widehat{z}(\varepsilon, \mu)=z_{*}(\mu)+O\left(\varepsilon \mu \ln \frac{1}{\varepsilon \mu}\right), \quad \varepsilon, \mu \rightarrow 0,
$$

где $z_{*}(\mu)$ - неподвижная точка (2.25) отображения (2.18). Подставим, далее, соотношение (2.52) вместе с равенством $\varphi=\widehat{\varphi}$ в асимптотические представления $(2.34),(2.42)$ и учтем, что функция $\mu x_{\widehat{\varphi}, \widehat{z}}(t, \mu)$, где $x_{\widehat{\varphi}, \widehat{z}}(t, \mu)$ - соответствующее решение релейного уравнения (2.4), с точностью до величин порядка $\varepsilon \mu \ln (1 /(\varepsilon \mu))$ совпадает с $\mu x_{*}(t, \mu)$ (см. (2.26)). В результате приходим к интересующим нас формулам (2.27).

2.3. Анализ вспомогательного линейного уравнения. В данном пункте объектом исследования является аналогичное (1.17) линейное уравнение

$$
\ddot{h}+2 \mu \dot{h}+h=\varkappa A(t, \varepsilon, \mu) h(t-\Delta)
$$

с запаздыванием $\Delta$, принадлежащим интервалу (2.2). Здесь, как и ранее, $\varepsilon, \mu>0$ - независимые малые параметры, $\varkappa$ - вспомогательный комплексный параметр, а коэффициент $A(t, \varepsilon, \mu)$ задан равенством

$$
A(t, \varepsilon, \mu)=\frac{1}{\varepsilon} F_{x}^{\prime}\left(\frac{x(t-\Delta, \varepsilon, \mu)}{\varepsilon}\right),
$$

где $x(t, \varepsilon, \mu)$ - периодическое решение уравнения (2.1), доставляемое теоремой 2.1 .

В связи с леммой 1.2 актуален вопрос об асимптотическом поведении при $\varepsilon, \mu \rightarrow 0$ мультипликаторов уравнения (2.53). Для его решения нам потребуются два вспомогательных утверждения. В первом из этих утверждений речь идет о необходимых в дальнейшем свойствах коэффициента (2.54). 
Для того чтобы сформулировать соответствующий результат, фиксируем произвольно $\sigma_{0} \in(0, \pi-\Delta)$ и обозначим через $\Sigma$ отрезок $\left[-\sigma_{0}, T(\varepsilon, \mu)-\sigma_{0}\right]$ с выброшенными интервалами

$$
(\Delta-\sqrt{\varepsilon \mu}, \Delta+\sqrt{\varepsilon \mu}), \quad\left(t_{1}(\varepsilon, \mu)+\Delta-\sqrt{\varepsilon \mu}, t_{1}(\varepsilon, \mu)+\Delta+\sqrt{\varepsilon \mu}\right) .
$$

Здесь, напомним, $T(\varepsilon, \mu)$ - период цикла $x(t, \varepsilon, \mu)$, а через $t_{1}(\varepsilon, \mu)$ обозначен корень уравнения $x(t, \varepsilon, \mu)=0$ из интервала $(0, T(\varepsilon, \mu))$. Заметим, что этот корень определяется однозначно и задается равенством $(2.38)$ при $(\varphi, z)=(\widehat{\varphi}, \widehat{z})$, где $(\widehat{\varphi}, \widehat{z})$ - неподвижная точка оператора (2.31). Тем самым в силу $(2.52)$ для него справедливо асимптотическое представление

$$
t_{1}(\varepsilon, \mu)=t_{*}(\mu)+O\left(\varepsilon \mu^{2} \ln \frac{1}{\varepsilon \mu}\right), \quad \varepsilon, \mu \rightarrow 0,
$$

где $t_{*}(\mu)$ - момент времени, фигурирующий в $(2.26)$.

Лемма 2.1. Найдутся такие достаточно малье $\varepsilon_{0}, \mu_{0}>0$ и такие независящие от $\varepsilon, \mu$ постоянные $M_{1}, M_{2}>0$, что при всех $0<\varepsilon \leqslant \varepsilon_{0}, 0<\mu \leqslant \mu_{0}$ выполняются неравенства

$$
\max _{-\sigma_{0} \leqslant t \leqslant \Delta-\sigma_{0}}|A(t, \varepsilon, \mu)| \leqslant M_{1} \varepsilon \mu^{2}, \quad \int_{\Sigma}|A(t, \varepsilon, \mu)| d t \leqslant M_{2} \sqrt{\varepsilon \mu^{3}} .
$$

Кроме того, справедливы асимптотические формуль

$$
\begin{gathered}
\int_{\Delta-\sqrt{\varepsilon \mu}}^{\Delta+\sqrt{\varepsilon \mu}} A(t, \varepsilon, \mu) d t=-\frac{2 \mu}{z_{*}(\mu)}+O\left(\sqrt{\varepsilon \mu^{3}}\right), \\
\int_{\Delta-\sqrt{\varepsilon \mu}}^{\Delta+\sqrt{\varepsilon \mu}}|A(t, \varepsilon, \mu)| d t=\frac{2 \mu}{z_{*}(\mu)}+O\left(\sqrt{\varepsilon \mu^{3}}\right), \\
\int_{t_{1}(\varepsilon, \mu)+\Delta-\sqrt{\varepsilon \mu}}^{t_{1}(\varepsilon, \mu)+\Delta+\sqrt{\varepsilon \mu}} A(t, \varepsilon, \mu) d t=-\frac{2 \mu}{z_{*}(\mu)}+O\left(\sqrt{\varepsilon \mu^{3}}\right), \\
\int_{t_{1}(\varepsilon, \mu)+\Delta-\sqrt{\varepsilon \mu}}^{t_{1}(\varepsilon, \mu)+\Delta+\sqrt{\varepsilon \mu}}|A(t, \varepsilon, \mu)| d t=\frac{2 \mu}{z_{*}(\mu)}+O\left(\sqrt{\varepsilon \mu^{3}}\right),
\end{gathered}
$$

где $z_{*}(\mu)-$ функиия (2.25).

ДокАЗАТЕЛЬСтво. Из свойств (1.7) функции $F(x)$ последовательно выводим неравенства

$$
\begin{gathered}
\left|F^{\prime}(x)\right| \leqslant \frac{M_{1}}{1+x^{2}}, \quad\left|F^{\prime \prime}(x)\right| \leqslant \frac{M_{2}}{1+|x|^{3}} \quad \forall x \in \mathbb{R}, \\
\left|F^{\prime}\left(x_{1}\right)-F^{\prime}\left(x_{2}\right)\right| \leqslant \frac{M_{3}}{1+\min \left(\left|x_{1}\right|^{3},\left|x_{2}\right|^{3}\right)}\left|x_{1}-x_{2}\right| \quad \forall x_{1}, x_{2} \in \mathbb{R} .
\end{gathered}
$$

Далее, объединяя оценку для $F^{\prime}(x)$ из (2.59) с асимптотическими свойствами периодического решения $x(t, \varepsilon, \mu)$ (см. $(2.27),(2.55))$, заключаем, что при $t \in \Sigma$

$$
|A(t, \varepsilon, \mu)| \leqslant \frac{M}{\varepsilon}\left(\frac{1}{1+(t-\Delta)^{2} /(\varepsilon \mu)^{2}}+\frac{1}{1+\left(t-t_{1}(\varepsilon, \mu)-\Delta\right)^{2} /(\varepsilon \mu)^{2}}\right) .
$$

Отсюда требуемые неравенства (2.56) вытекают очевидным образом. 
Обратимся теперь к асимптотическим представлениям (2.57), (2.58) и докажем, к примеру, (2.57) (поскольку (2.58) обосновывается аналогично). С этой целью перейдем на промежутке $\Delta-\sqrt{\varepsilon \mu} \leqslant t \leqslant \Delta+\sqrt{\varepsilon \mu}$ к переменной $\tau$, $|\tau| \leqslant 1 / \sqrt{\varepsilon \mu}$, где $\tau=(t-\Delta) /(\varepsilon \mu)$. Далее, разложим функцию $x(\varepsilon \mu \tau, \varepsilon, \mu) / \varepsilon$ по формуле Тейлора в точке $\tau=0$. В результате, принимая во внимание свойство $x(0, \varepsilon, \mu) \equiv 0$, получаем представление

$$
\frac{x(\varepsilon \mu \tau, \varepsilon, \mu)}{\varepsilon}=\mu \dot{x}(\varepsilon \mu \bar{\tau}, \varepsilon, \mu) \tau,
$$

где значение $\bar{\tau}$ таково, что $|\bar{\tau}| \leqslant|\tau|$.

Последующий анализ базируется на вытекающих из (2.26), (2.27) соотношениях

$$
\begin{gathered}
\mu \dot{x}(\varepsilon \mu \tau, \varepsilon, \mu)=\mu \dot{x}_{*}(\varepsilon \mu \tau, \mu)+O\left(\varepsilon \mu \ln \frac{1}{\varepsilon \mu}\right), \quad-\frac{1}{\sqrt{\varepsilon \mu}} \leqslant \tau \leqslant \frac{1}{\sqrt{\varepsilon \mu}}, \\
\mu \dot{x}_{*}(0, \mu)=z_{*}(\mu),
\end{gathered}
$$

где $z_{*}(\mu)$ - функция (2.25). Учитывая эти формулы в правой части из представления (2.60), приходим к выводу, что

$$
\frac{x(\varepsilon \mu \tau, \varepsilon, \mu)}{\varepsilon}=\left(z_{*}(\mu)+O(\sqrt{\varepsilon \mu})\right) \tau,
$$

где остаток равномерен по $\tau \in[-1 / \sqrt{\varepsilon \mu}, 1 / \sqrt{\varepsilon \mu}]$.

На заключительном этапе воспользуемся третьим неравенством (2.59), из которого в силу (2.61) имеем

$$
\left|F^{\prime}\left(\frac{x(\varepsilon \mu \tau, \varepsilon, \mu)}{\varepsilon}\right)-F^{\prime}\left(z_{*}(\mu) \tau\right)\right| \leqslant \frac{M|\tau|}{1+|\tau|^{3}} \sqrt{\varepsilon \mu} \quad \forall \tau \in\left[-\frac{1}{\sqrt{\varepsilon \mu}}, \frac{1}{\sqrt{\varepsilon \mu}}\right] .
$$

Используем, далее, оценку (2.62) вместе со свойствами (1.6), (1.7) функции $F(x)$ для вычисления интегралов из (2.57). В результате убеждаемся в том, чTО

$$
\begin{gathered}
\int_{\Delta-\sqrt{\varepsilon \mu}}^{\Delta+\sqrt{\varepsilon \mu}} A(t, \varepsilon, \mu) d t=\mu \int_{-1 / \sqrt{\varepsilon \mu}}^{1 / \sqrt{\varepsilon \mu}} F^{\prime}\left(z_{*}(\mu) \tau\right) d \tau+O\left(\sqrt{\varepsilon \mu^{3}}\right) \\
=\mu \int_{-\infty}^{+\infty} F^{\prime}\left(z_{*}(\mu) \tau\right) d \tau+O\left(\sqrt{\varepsilon \mu^{3}}\right)=-\frac{2 \mu}{z_{*}(\mu)}+O\left(\sqrt{\varepsilon \mu^{3}}\right) \\
\int_{\Delta-\sqrt{\varepsilon \mu}}^{\Delta+\sqrt{\varepsilon \mu}}|A(t, \varepsilon, \mu)| d t=\mu \int_{-1 / \sqrt{\varepsilon \mu}}^{1 / \sqrt{\varepsilon \mu}}\left|F^{\prime}\left(z_{*}(\mu) \tau\right)\right| d \tau+O\left(\sqrt{\varepsilon \mu^{3}}\right) \\
=\mu \int_{-\infty}^{+\infty}\left|F^{\prime}\left(z_{*}(\mu) \tau\right)\right| d \tau+O\left(\sqrt{\varepsilon \mu^{3}}\right)=\frac{2 \mu}{z_{*}(\mu)}+O\left(\sqrt{\varepsilon \mu^{3}}\right) .
\end{gathered}
$$

Лемма доказана.

Перед формулировкой следующего утверждения введем в рассмотрение банахово пространство $C_{0}$ непрерывных на отрезке $-\Delta-\sigma_{0} \leqslant t \leqslant-\sigma_{0}$ комплекснозначных функций $\varphi_{0}(t), \varphi_{0}\left(-\sigma_{0}\right)=0$, с нормой

$$
\left\|\varphi_{0}\right\|=\max _{-\Delta-\sigma_{0} \leqslant t \leqslant-\sigma_{0}}\left|\varphi_{0}(t)\right| .
$$


Обозначим, далее, через $h_{\varphi_{0}}(t, \varepsilon, \mu, \varkappa), t \geqslant-\sigma_{0}$, решение уравнения $(2.53)$ с начальными условиями $h=\varphi_{0}(t),-\Delta-\sigma_{0} \leqslant t \leqslant-\sigma_{0},\left.\dot{h}\right|_{t=-\sigma_{0}}=0$, где $\varphi_{0}(t)-$ произвольный элемент пространства $C_{0}$.

ЛЕмма 2.2. Для каждого $r>0$ можно указать такие положительные постоянные $M=M(r), \varepsilon_{0}=\varepsilon_{0}(r), \mu_{0}=\mu_{0}(r)$, что при всех $0<\varepsilon \leqslant \varepsilon_{0}$, $0<\mu \leqslant \mu_{0}, \varkappa \in B(r) \stackrel{\text { def }}{=}\{\varkappa \in \mathbb{C}:|\varkappa| \leqslant r\}$ и при любом $\varphi_{0} \in C_{0}$ имеет место оценка

$$
\max _{-\sigma_{0} \leqslant t \leqslant T(\varepsilon, \mu)-\sigma_{0}}\left(\left|h_{\varphi_{0}}\right|+\left|\dot{h}_{\varphi_{0}}\right|+\left|\frac{\partial}{\partial \varkappa} h_{\varphi_{0}}\right|+\left|\frac{\partial}{\partial \varkappa} \dot{h}_{\varphi_{0}}\right|\right) \leqslant M \varepsilon \mu^{2}\left\|\varphi_{0}\right\| .
$$

ДокАЗАТЕЛЬСтво. Фиксируем произвольно положительное $r$ и будем считать, что $\varkappa \in B(r)$. Обратимся, далее, к отрезку $-\sigma_{0} \leqslant t \leqslant \Delta-\sigma_{0}$, на котором для коэффициента $A(t, \varepsilon, \mu)$ справедлива первая оценка $(2.56)$, а для решения $h_{\varphi_{0}}(t, \varepsilon, \mu, \varkappa)-$ явная формула

$$
h_{\varphi_{0}}(t, \varepsilon, \mu, \varkappa)=\varkappa \int_{-\sigma_{0}}^{t} K(t-\tau, \mu) A(\tau, \varepsilon, \mu) \varphi_{0}(\tau-\Delta) d \tau, \quad-\sigma_{0} \leqslant t \leqslant \Delta-\sigma_{0},
$$

где $K(t, \mu)$ - функция из (2.32). Объединяя эти факты, сначала убеждаемся в аналитичности $h_{\varphi_{0}}$ и $\dot{h}_{\varphi_{0}}$ по переменной $\varkappa \in B(r)$ на данном промежутке изменения $t$, а затем - в справедливости неравенства

$$
\max _{t}\left(\left|h_{\varphi_{0}}\right|+\left|\dot{h}_{\varphi_{0}}\right|+\left|\frac{\partial}{\partial \varkappa} h_{\varphi_{0}}\right|+\left|\frac{\partial}{\partial \varkappa} \dot{h}_{\varphi_{0}}\right|\right) \leqslant M \varepsilon \mu^{2}\left\|\varphi_{0}\right\| .
$$

Для распространения оценки (2.64) на оставшийся промежуток времени $\left[\Delta-\sigma_{0}, T(\varepsilon, \mu)-\sigma_{0}\right]$ воспользуемся методом шагов. А именно, разобьем данный промежуток на отрезки $\left[\Delta-\sigma_{0}+l \Delta, 2 \Delta-\sigma_{0}+l \Delta\right], l=0,1, \ldots, l_{0}$, и $\left[2 \Delta-\sigma_{0}+l_{0} \Delta\right.$, $\left.T(\varepsilon, \mu)-\sigma_{0}\right]$, где $l_{0}=\lfloor(T(\varepsilon, \mu)-2 \Delta) / \Delta\rfloor,\lfloor\cdot\rfloor-$ целая часть. Заметим, далее, что на $l$-м отрезке изменения $t$ решение $h_{\varphi_{0}}(t, \varepsilon, \mu, \varkappa)$ задается равенством

$$
\begin{array}{r}
h_{\varphi_{0}}(t, \varepsilon, \mu, \varkappa)=\varkappa \int_{\Delta-\sigma_{0}+l \Delta}^{t} K(t-\tau, \mu) A(\tau, \varepsilon, \mu) h_{\varphi_{0}}(\tau-\Delta, \varepsilon, \mu, \varkappa) d \tau \\
+\exp \left(-\mu\left(t-\Delta+\sigma_{0}-l \Delta\right)\right)\left[c_{1} \cos \left(\omega\left(t-\Delta+\sigma_{0}-l \Delta\right)\right)\right. \\
\left.+c_{2} \sin \left(\omega\left(t-\Delta+\sigma_{0}-l \Delta\right)\right)\right],
\end{array}
$$

где

$$
c_{1}=h_{\varphi_{0}}\left(\Delta-\sigma_{0}+l \Delta, \varepsilon, \mu, \varkappa\right), \quad \omega c_{2}-\mu c_{1}=\dot{h}_{\varphi_{0}}\left(\Delta-\sigma_{0}+l \Delta, \varepsilon, \mu, \varkappa\right) .
$$

Последующий способ действий стандартен. Сначала, опираясь на формулы $(2.65),(2.66)$ и уже установленную аналитичность функций $h_{\varphi_{0}}, \dot{h}_{\varphi_{0}}$ по $\varkappa$ на $(l-1)$-м отрезке, убеждаемся в справедливости этого свойства на $l$-м отрезке. Затем, учитывая факт интегральной ограниченности

$$
\int_{-\sigma_{0}}^{T(\varepsilon, \mu)-\sigma_{0}}|A(t, \varepsilon, \mu)| d t \leqslant M \mu, \quad M=\text { const }>0
$$

имеющий место в силу (2.56)-(2.58), замечаем, что из $(2.65),(2.66)$ и из уже известной оценки вида $(2.64)$ на $(l-1)$-м шаге вытекает требуемая оценка на $l$-м шаге изменения $t$. Лемма доказана. 
Перейдем теперь непосредственно к интересующему нас вопросу об асимптотическом вычислении мультипликаторов уравнения (2.53). С этой целью рассмотрим оператор монодромии $U(\varepsilon, \mu, \varkappa)$ данного уравнения, действующий в пространстве $C\left[-\Delta-\sigma_{0},-\sigma_{0}\right] \times \mathbb{C}$ (над полем комплексных чисел с нормой $\left.\|(\varphi, z)\|=\max _{-\Delta-\sigma_{0} \leqslant t \leqslant-\sigma_{0}}|\varphi(t)|+|z|\right)$ по правилу

$$
\begin{gathered}
U(\varepsilon, \mu, \varkappa)[(\varphi, z)]=\left(h_{\varphi, z}(t+T(\varepsilon, \mu), \varepsilon, \mu, \varkappa), \dot{h}_{\varphi, z}\left(T(\varepsilon, \mu)-\sigma_{0}, \varepsilon, \mu, \varkappa\right)\right), \\
-\Delta-\sigma_{0} \leqslant t \leqslant-\sigma_{0},
\end{gathered}
$$

где $h=h_{\varphi, z}(t, \varepsilon, \mu, \varkappa),-\sigma_{0} \leqslant t \leqslant T(\varepsilon, \mu)-\sigma_{0},-$ решение уравнения (2.53) с начальными условиями $h=\varphi(t)$ при $-\Delta-\sigma_{0} \leqslant t \leqslant-\sigma_{0},\left.\dot{h}\right|_{t=-\sigma_{0}}=z$. Далее, обозначим через $\nu_{s}(\varepsilon, \mu, \varkappa), s \in \mathbb{N}$, собственные значения оператора (2.67), занумерованные в порядке убывания модулей. Справедливо следующее утверждение.

Теорема 2.2. Для любого $r>0$ найдутся такие положительные постоянные $\varepsilon_{0}=\varepsilon_{0}(r), \mu_{0}=\mu_{0}(r), M=M(r)$, ито при всех $0<\varepsilon \leqslant \varepsilon_{0}, 0<\mu \leqslant \mu_{0}$ $u \varkappa \in B(r)$ выполняется неравенство

$$
\sup _{s \geqslant 3}\left|\nu_{s}(\varepsilon, \mu, \varkappa)\right| \leqslant M \varepsilon \mu^{2} .
$$

Оставшиеся мультипликаторы $\nu_{j}(\varepsilon, \mu, \varkappa), j=1,2$, допускают при $\varepsilon, \mu \rightarrow 0$ равномерные по $\varkappa \in B(r)$ асимптотические представления

$$
\nu_{1}(\varepsilon, \mu, \varkappa)=1+\mu \lambda_{+}(\varkappa)+o(\mu), \quad \nu_{2}(\varepsilon, \mu, \varkappa)=1+\mu \lambda_{-}(\varkappa)+o(\mu),
$$

где

$$
\lambda_{ \pm}(\varkappa)=\pi\left[\varkappa-2 \pm \sqrt{(\varkappa-2)^{2}+\frac{4(\varkappa-1)}{\sin ^{2} \Delta}}\right] .
$$

ДокАЗАтЕЛьСтво. Как и при обосновании леммы 2.2 , фиксируем произвольно $r>0$ и предполагаем, что комплексный параметр $\varkappa$ пробегает шар $B(r)$. Далее, введем в рассмотрение конечномерный оператор

$$
\begin{aligned}
& V(\varepsilon, \mu, \varkappa)[(\varphi, z)]=\varphi\left(-\sigma_{0}\right)\left(h_{1}(t+T(\varepsilon, \mu), \varepsilon, \mu, \varkappa), \dot{h}_{1}\left(T(\varepsilon, \mu)-\sigma_{0}, \varepsilon, \mu, \varkappa\right)\right) \\
& \quad+z\left(h_{2}(t+T(\varepsilon, \mu), \varepsilon, \mu, \varkappa), \dot{h}_{2}\left(T(\varepsilon, \mu)-\sigma_{0}, \varepsilon, \mu, \varkappa\right)\right), \quad-\Delta-\sigma_{0} \leqslant t \leqslant-\sigma_{0},
\end{aligned}
$$

где через $h_{j}(t, \varepsilon, \mu, \varkappa), j=1,2$, обозначены решения уравнения (2.53) на отрезке $-\sigma_{0} \leqslant t \leqslant T(\varepsilon, \mu)-\sigma_{0}$ с начальными условиями $h_{1} \equiv 1$ при $-\Delta-\sigma_{0} \leqslant t \leqslant-\sigma_{0}$, $\left.\dot{h}_{1}\right|_{t=-\sigma_{0}}=0$ и $h_{2} \equiv 0$ при $-\Delta-\sigma_{0} \leqslant t \leqslant-\sigma_{0},\left.\dot{h}_{2}\right|_{t=-\sigma_{0}}=1$.

Рассмотрим вопрос о связи между операторами (2.67) и (2.71). С этой целью обратим внимание на то, что фигурирующее в $(2.67)$ решение $h_{\varphi, z}(t, \varepsilon, \mu, \varkappa)$ может быть представлено в виде

$$
h_{\varphi, z}(t, \varepsilon, \mu, \varkappa)=\varphi\left(-\sigma_{0}\right) h_{1}(t, \varepsilon, \mu, \varkappa)+z h_{2}(t, \varepsilon, \mu, \varkappa)+h_{\varphi_{0}}(t, \varepsilon, \mu, \varkappa),
$$

где $h_{\varphi_{0}}$ - решение уравнения $(2.53)$ с начальными условиями

$$
h=\varphi_{0}(t) \stackrel{\text { def }}{=} \varphi(t)-\varphi\left(-\sigma_{0}\right) \in C_{0}, \quad-\Delta-\sigma_{0} \leqslant t \leqslant-\sigma_{0},\left.\quad \dot{h}\right|_{t=-\sigma_{0}}=0 .
$$


Таким образом, третье слагаемое из (2.72) в силу леммы 2.1 удовлетворяет оценке (2.63). Отсюда, в свою очередь, следует, что для оператора $W(\varepsilon, \mu, \varkappa)=$ $U(\varepsilon, \mu, \varkappa)-V(\varepsilon, \mu, \varkappa)$ справедливо неравенство

$$
\begin{aligned}
& \|W(\varepsilon, \mu, \varkappa)\|_{C\left[-\Delta-\sigma_{0},-\sigma_{0}\right] \times \mathbb{C} \rightarrow C\left[-\Delta-\sigma_{0},-\sigma_{0}\right] \times \mathbb{C}} \\
& +\left\|\frac{\partial}{\partial \varkappa} W(\varepsilon, \mu, \varkappa)\right\|_{C\left[-\Delta-\sigma_{0},-\sigma_{0}\right] \times \mathbb{C} \rightarrow C\left[-\Delta-\sigma_{0},-\sigma_{0}\right] \times \mathbb{C}} \leqslant M \varepsilon \mu^{2} .
\end{aligned}
$$

На следующем этапе доказательства изучим спектральные свойства оператора (2.71). Нетрудно увидеть, что его спектр состоит из собственных значений двумерной матрицы

$$
\mathscr{D}=\left.\left(\begin{array}{ll}
h_{1}(t, \varepsilon, \mu, \varkappa) & h_{2}(t, \varepsilon, \mu, \varkappa) \\
\dot{h}_{1}(t, \varepsilon, \mu, \varkappa) & \dot{h}_{2}(t, \varepsilon, \mu, \varkappa)
\end{array}\right)\right|_{t=T(\varepsilon, \mu)-\sigma_{0}}
$$

и из нулевого собственного значения бесконечной кратности. Таким образом, проблема спектрального анализа оператора (2.71) сводится к асимптотическому вычислению матрицы (2.74).

Для решения поставленной проблемы дополним уравнение (2.53) начальными условиями

$$
\begin{aligned}
& h \equiv 1 \quad \text { при } \quad-\Delta-\sigma_{0} \leqslant t \leqslant-\sigma_{0},\left.\quad \dot{h}\right|_{t=-\sigma_{0}}=0 \text {, } \\
& h \equiv 0 \quad \text { при } \quad-\Delta-\sigma_{0} \leqslant t \leqslant-\sigma_{0},\left.\quad \dot{h}\right|_{t=-\sigma_{0}}=1
\end{aligned}
$$

соответственно и проинтегрируем его на отрезке $-\sigma_{0} \leqslant t \leqslant T(\varepsilon, \mu)-\sigma_{0}$ методом шагов, учитывая $\delta$-образное поведение коэффициента $A(t, \varepsilon, \mu)$ (см. формулы (2.56)-(2.58)). В результате убеждаемся в том, что, во-первых, функции $h_{j}(t, \varepsilon, \mu, \varkappa), \dot{h}_{j}(t, \varepsilon, \mu, \varkappa), j=1,2$, аналитически зависят от $\varkappa \in B(r)$ и допускают оценки вида

$$
\max _{-\sigma_{0} \leqslant t \leqslant T(\varepsilon, \mu)-\sigma_{0}}\left(\left|h_{j}\right|+\left|\dot{h}_{j}\right|+\left|\frac{\partial}{\partial \varkappa} h_{j}\right|+\left|\frac{\partial}{\partial \varkappa} \dot{h}_{j}\right|\right) \leqslant M, \quad j=1,2 ;
$$

во-вторых, при $\varepsilon, \mu \rightarrow 0$ равномерно по $t \in \Sigma, \varkappa \in B(r)$

$$
\begin{gathered}
h_{j}=h_{j, 0}(t, \mu, \varkappa)+O\left(\sqrt{\varepsilon \mu^{3}}\right), \quad \dot{h}_{j}=\dot{h}_{j, 0}(t, \mu, \varkappa)+O\left(\sqrt{\varepsilon \mu^{3}}\right), \\
\frac{\partial}{\partial \varkappa} h_{j}=\frac{\partial}{\partial \varkappa} h_{j, 0}(t, \mu, \varkappa)+O\left(\sqrt{\varepsilon \mu^{3}}\right), \quad \frac{\partial}{\partial \varkappa} \dot{h}_{j}=\frac{\partial}{\partial \varkappa} \dot{h}_{j, 0}(t, \mu, \varkappa)+O\left(\sqrt{\varepsilon \mu^{3}}\right),
\end{gathered}
$$

где $h_{j, 0}(t, \mu, \varkappa), j=1,2,-$ решения импульсной задачи Коши

$$
\begin{gathered}
\ddot{h}+2 \mu \dot{h}+h=0, \\
h(\Delta+0)=h(\Delta-0), \quad \dot{h}(\Delta+0)=\dot{h}(\Delta-0)-\frac{2 \varkappa \mu}{z_{*}(\mu)} h(0), \\
h\left(\Delta+t_{*}(\mu)+0\right)=h\left(\Delta+t_{*}(\mu)-0\right), \\
\dot{h}\left(\Delta+t_{*}(\mu)+0\right)=\dot{h}\left(\Delta+t_{*}(\mu)-0\right)-\frac{2 \varkappa \mu}{z_{*}(\mu)} h\left(t_{*}(\mu)\right)
\end{gathered}
$$


с начальными условиями $(h, \dot{h})_{t=-\sigma_{0}}=(1,0)$ и $\left.(h, \dot{h})\right|_{t=-\sigma_{0}}=(0,1)$ соответственно.

Перечисленные факты сводят интересующий нас вопрос об асимптотическом поведении собственных значений матрицы (2.74) к построению асимптотики по $\mu$ введенных выше решений $h_{j, 0}(t, \mu, \varkappa), j=1,2$. Соответствующий анализ, связанный с интегрированием системы (2.77), не вызывает затруднений. Поэтому здесь ограничимся лишь следующими итоговыми формулами:

$$
\begin{gathered}
h_{1,0}=\cos \left(t+\sigma_{0}\right)+\mu\left[\sin \left(t+\sigma_{0}\right)-\left(t+\sigma_{0}\right) \cos \left(t+\sigma_{0}\right)\right]+O\left(\mu^{2}\right), \\
h_{2,0}=\sin \left(t+\sigma_{0}\right)-\mu\left(t+\sigma_{0}\right) \sin \left(t+\sigma_{0}\right)+O\left(\mu^{2}\right)
\end{gathered}
$$

при $0 \leqslant t \leqslant \Delta$;

$$
\begin{aligned}
& h_{1,0}=\cos \left(t+\sigma_{0}\right)+\mu\left[\sin \left(t+\sigma_{0}\right)-\right.\left(t+\sigma_{0}\right) \cos \left(t+\sigma_{0}\right) \\
&\left.-\frac{\pi \varkappa}{\sin \Delta} \sin (t-\Delta) \cos \sigma_{0}\right]+O\left(\mu^{2}\right), \\
& h_{2,0}=\sin \left(t+\sigma_{0}\right)+\mu\left[-\left(t+\sigma_{0}\right) \sin \left(t+\sigma_{0}\right)\right. \\
&\left.-\frac{\pi \varkappa}{\sin \Delta} \sin (t-\Delta) \sin \sigma_{0}\right]+O\left(\mu^{2}\right)
\end{aligned}
$$

при $\Delta \leqslant t \leqslant \Delta+t_{*}(\mu)$;

$$
\begin{gathered}
h_{1,0}=\cos \left(t+\sigma_{0}\right)+\mu\left[\sin \left(t+\sigma_{0}\right)-\left(t+\sigma_{0}\right) \cos \left(t+\sigma_{0}\right)-\frac{\pi \varkappa}{\sin \Delta} \sin (t-\Delta) \cos \sigma_{0}\right. \\
\left.-\frac{\pi \varkappa}{\sin \Delta} \sin \left(t-\Delta-t_{*}(\mu)\right) \cos \left(t_{*}(\mu)+\sigma_{0}\right)\right]+O\left(\mu^{2}\right), \\
h_{2,0}=\sin \left(t+\sigma_{0}\right)+\mu\left[-\left(t+\sigma_{0}\right) \sin \left(t+\sigma_{0}\right)-\frac{\pi \varkappa}{\sin \Delta} \sin (t-\Delta) \sin \sigma_{0}\right. \\
\left.-\frac{\pi \varkappa}{\sin \Delta} \sin \left(t-\Delta-t_{*}(\mu)\right) \sin \left(t_{*}(\mu)+\sigma_{0}\right)\right]+O\left(\mu^{2}\right)
\end{gathered}
$$

при $\Delta+t_{*}(\mu) \leqslant t \leqslant 2 t_{*}(\mu)$.

Добавим еще, что все асимптотические равенства (2.78)-(2.80), а также аналогичные равенства для $\dot{h}_{j, 0}, \partial h_{j, 0} / \partial \varkappa, \partial \dot{h}_{j, 0} / \partial \varkappa, j=1,2$, справедливы равномерно по $\varkappa \in B(r)$ и по $t$ из соответствующих промежутков.

Итак, теперь у нас есть все необходимое для анализа собственных значений матрицы (2.74). Действительно, объединяя асимптотические представления (2.76)-(2.80) с асимптотикой периода $T(\varepsilon, \mu)($ см. $(2.26),(2.27))$, приходим к выводу, что характеристическое уравнение $\operatorname{det}(\mathscr{D}-\lambda I)=0$ этой матрицы после замены $\lambda=1+\mu \widetilde{\lambda}$ и сокращения на $\mu^{2}$ записывается в виде

$$
\widetilde{\lambda}^{2}+p(\varepsilon, \mu, \varkappa) \widetilde{\lambda}+q(\varepsilon, \mu, \varkappa)=0,
$$

где

$$
\begin{array}{cc}
p=2 \pi(2-\varkappa)+O(\sqrt{\varepsilon \mu}+\mu), \quad \frac{\partial p}{\partial \varkappa}=-2 \pi+O(\sqrt{\varepsilon \mu}+\mu), \\
q=\frac{4 \pi^{2}}{\sin ^{2} \Delta}(1-\varkappa)+O(\sqrt{\varepsilon \mu}+\mu), & \frac{\partial q}{\partial \varkappa}=-\frac{4 \pi^{2}}{\sin ^{2} \Delta}+O(\sqrt{\varepsilon \mu}+\mu) .
\end{array}
$$

4 Серия математическая, т. 78, № 4 
Отсюда несложно вывести, что для собственных значений $\lambda_{j}(\varepsilon, \mu, \varkappa), j=1,2$, матрицы (2.74) имеют место равномерные по $\varkappa \in B(r)$ асимптотические представления

$$
\lambda_{1}=1+\mu \lambda_{+}(\varkappa)+o(\mu), \quad \lambda_{2}=1+\mu \lambda_{-}(\varkappa)+o(\mu), \quad \varepsilon, \mu \rightarrow 0,
$$

где $\lambda_{ \pm}(\varkappa)$ - функции $(2.70)$.

Обратимся теперь к исходному оператору $U$ и заметим, что в силу равенств $U=V+W,(\lambda I-U)^{-1}=\left(I-(\lambda I-V)^{-1} W\right)^{-1}(\lambda I-V)^{-1}$ любое значение $\lambda \in \mathbb{C}$, для которого

$$
\left\|(\lambda I-V)^{-1} W\right\|_{C\left[-\Delta-\sigma_{0},-\sigma_{0}\right] \times \mathbb{C} \rightarrow C\left[-\Delta-\sigma_{0},-\sigma_{0}\right] \times \mathbb{C}<1,}
$$

принадлежит резольвентному множеству этого оператора. Напомним, далее, что оператор $W$ допускает оценку вида (2.73). В случае оператора $(\lambda I-V)^{-1}$, опираясь на соотношения

$$
\begin{aligned}
& (\lambda I-V)^{-1}[(\varphi, z)]= \\
& =\frac{1}{\lambda}(\varphi(t), z)+c_{1}\left(h_{1}(t+T(\varepsilon, \mu), \varepsilon, \mu, \varkappa), \dot{h}_{1}\left(T(\varepsilon, \mu)-\sigma_{0}, \varepsilon, \mu, \varkappa\right)\right) \\
& \quad+c_{2}\left(h_{2}(t+T(\varepsilon, \mu), \varepsilon, \mu, \varkappa), \dot{h}_{2}\left(T(\varepsilon, \mu)-\sigma_{0}, \varepsilon, \mu, \varkappa\right)\right), \quad-\Delta-\sigma_{0} \leqslant t \leqslant-\sigma_{0}, \\
& \quad\left(\begin{array}{c}
c_{1} \\
c_{2}
\end{array}\right)=\frac{1}{\lambda}(\lambda I-\mathscr{D})^{-1}\left(\begin{array}{c}
\varphi\left(-\sigma_{0}\right) \\
z
\end{array}\right)
\end{aligned}
$$

и оценки (2.75), получаем неравенство

$$
\begin{gathered}
\left\|(\lambda I-V)^{-1}\right\|_{C\left[-\Delta-\sigma_{0},-\sigma_{0}\right] \times \mathbb{C} \rightarrow C\left[-\Delta-\sigma_{0},-\sigma_{0}\right] \times \mathbb{C}} \leqslant \frac{M\left(1+|\lambda|^{2}\right)}{|\lambda|\left|\lambda-\lambda_{1}\right|\left|\lambda-\lambda_{2}\right|} \\
\forall \lambda \in \mathbb{C}, \quad \lambda \neq 0, \lambda_{1}, \lambda_{2},
\end{gathered}
$$

где, напомним, $\lambda_{1}, \lambda_{2}$ - собственные значения матрицы (2.74).

На завершающем этапе доказательства объединим оценки (2.73), (2.85) с асимптотическими представлениями (2.83). В результате убеждаемся в том, что любая точка $\lambda \in \mathbb{C}$ из множества

$$
\mathbb{C} \backslash\left\{O_{1} \cup O_{2} \cup O_{3}\right\},
$$

где

$$
\begin{gathered}
O_{1}=\left\{\lambda:|\lambda|<M_{1} \varepsilon \mu^{2}\right\}, \quad O_{2}=\left\{\lambda:\left|\lambda-\lambda_{1}\right|<M_{2} \sqrt{\varepsilon} \mu\right\}, \\
O_{3}=\left\{\lambda:\left|\lambda-\lambda_{2}\right|<M_{3} \sqrt{\varepsilon} \mu\right\},
\end{gathered}
$$

а постоянные $M_{j}, j=1,2,3$, подходящим образом велики, удовлетворяет условию (2.84) и, следовательно, является регулярной для оператора $U$. Спектр этого оператора заведомо принадлежит шарам (2.87). Отсюда и из (2.83) требуемые соотношения (2.68), (2.69) вытекают очевидным образом. Теорема доказана.

Приведем некоторые следствия из теоремы 2.2. В первую очередь обратим внимание на то, что в силу (2.82) корни уравнения (2.81) становятся кратными при значениях $\varkappa=\varkappa_{*, j}(\varepsilon, \mu), j=1,2$, где

$$
\varkappa_{*, j}(\varepsilon, \mu)=\varkappa_{*, j}^{0}+O(\sqrt{\varepsilon \mu}+\mu), \quad j=1,2,
$$


а $\varkappa_{*, j}^{0}, j=1,2,-$ корни уравнения

$$
(\varkappa-2)^{2}+\frac{4}{\sin ^{2} \Delta}(\varkappa-1)=0 .
$$

Именно по этой причине в $(2.86),(2.87)$ были рассмотрены окрестности точек $\lambda=\lambda_{j}, j=1,2$, порядка $\sqrt{\varepsilon} \mu$, а не $\varepsilon \mu^{2}$, как в случае $\lambda=0$. Если предположить, что $\varkappa \in B_{\delta}(r)$, где $B_{\delta}(r)$ - шар $B(r)$ с выброшенными множествами $\{\varkappa \in$ $\left.\mathbb{C}:\left|\varkappa-\varkappa_{*, j}(\varepsilon, \mu)\right|<\delta\right\}, j=1,2, \delta=$ const $>0$, то справедливо следующее утверждение.

СЛЕДСТВИЕ 2.1. Пусть в условиях теоремы 2.2 параметр х принадлежит множеству $B_{\delta}(r)$. Тогда мультипликаторы (2.69) являются простыми, аналитически зависящими от и и допускают асимптотику

$$
\begin{array}{cc}
\nu_{1}(\varepsilon, \mu, \varkappa) & =1+\mu \lambda_{+}(\varkappa)+O\left(\sqrt{\varepsilon \mu^{3}}+\mu^{2}\right), \\
\nu_{2}(\varepsilon, \mu, \varkappa) & =1+\mu \lambda_{-}(\varkappa)+O\left(\sqrt{\varepsilon \mu^{3}}+\mu^{2}\right), \\
\frac{\partial \nu_{1}}{\partial \varkappa}=\mu \lambda_{+}^{\prime}(\varkappa)+O\left(\sqrt{\varepsilon \mu^{3}}+\mu^{2}\right), & \frac{\partial \nu_{2}}{\partial \varkappa}=\mu \lambda_{-}^{\prime}(\varkappa)+O\left(\sqrt{\varepsilon \mu^{3}}+\mu^{2}\right) .
\end{array}
$$

ДоказАтельство. Заметим следующее. Во-первых, при $\varkappa \in B_{\delta}(r)$ корни уравнения (2.81) заведомо простые, а значит, аналитически зависят от параметра $\varkappa$. Более того, из (2.82) вытекает, что для собственных значений $\lambda_{j}(\varepsilon, \mu, \varkappa)$, $j=1,2$, матрицы $(2.74)$ в этом случае имеют место представления $(2.83)$ с остатками порядка $O\left(\sqrt{\varepsilon \mu^{3}}+\mu^{2}\right)$ и данные представления сохраняются при дифференцировании по $\varkappa$. Во-вторых, при возмущении оператора $V$ аналитической по $\varkappa$ добавкой $W$ порядка $\varepsilon \mu^{2}$ собственные значения $\lambda_{j}(\varepsilon, \mu, \varkappa), j=1,2$, переходят в простые и аналитические по $\varkappa$ собственные значения $\nu_{j}(\varepsilon, \mu, \varkappa)$, $j=1,2$, причем

$$
\nu_{j}=\lambda_{j}+O\left(\varepsilon \mu^{2}\right), \quad \frac{\partial \nu_{j}}{\partial \varkappa}=\frac{\partial \lambda_{j}}{\partial \varkappa}+O\left(\varepsilon \mu^{2}\right), \quad j=1,2 .
$$

Отсюда, в свою очередь, следует, что имеют место требуемые представления (2.90). Следствие доказано.

СледСтвиЕ 2.2. Справедливы соотношения

$$
\begin{gathered}
\left.\nu_{1}(\varepsilon, \mu, \varkappa)\right|_{\varkappa=1} \equiv 1,\left.\quad \frac{\partial \nu_{1}}{\partial \varkappa}\right|_{\varkappa=1}=\frac{2 \pi}{\sin ^{2} \Delta} \mu+O\left(\sqrt{\varepsilon \mu^{3}}+\mu^{2}\right), \\
\left.\nu_{2}(\varepsilon, \mu, \varkappa)\right|_{\varkappa=1}=1-2 \pi \mu+O\left(\sqrt{\varepsilon \mu^{3}}+\mu^{2}\right) .
\end{gathered}
$$

ДокАЗАТЕЛЬСтво. Действительно, поскольку точка $\varkappa=1$ не является корнем уравнения (2.89), она заведомо принадлежит множеству $B_{\delta}(r)$ при подходящем выборе постоянных $r, \delta>0$. Тем самым, при $\varkappa=1$ мы можем воспользоваться равенствами (2.90), из которых второе и третье соотношения (2.91) вытекают очевидным образом.

Для обоснования первого свойства (2.91) достаточно заметить, что при $\varkappa=1$ уравнение (2.53) представляет собой уравнение в вариациях на цикле $x(t, \varepsilon, \mu)$, о котором идет речь в теореме 2.1. Следовательно, оно с необходимостью 
имеет единичный мультипликатор. Более того, опираясь на (2.68), (2.91), теперь мы можем утверждать, что, во-первых, упомянутый цикл экспоненциально орбитально устойчив, во-вторых, отвечающая ему неподвижная точка $(\widehat{\varphi}, \widehat{z}) \in \Omega\left(q_{1}, q_{2}, z_{1}, z_{2}\right)$ оператора (2.31) единственна (ранее, напомним, мы установили только факт ее существования).

\section{§ 3. Итоговые результаты}

3.1. Теоремы о существовании и устойчивости бегущих волн. Обратимся к исходной системе (1.8) и напомним, что в силу леммы 1.1 проблема существования ее бегущих волн сводится к отысканию периодических решений вспомогательного уравнения (2.1), имеющих периоды $m \Delta / k, k \in \mathbb{N}$. В связи с этим в дальнейшем периодическое решение уравнения (2.1), доставляемое теоремой 2.1 , и его период будем обозначать через $x(t, \varepsilon, \mu, \Delta)$ и $T(\varepsilon, \mu, \Delta)$ соответственно, подчеркивая явно зависимость указанных функций от $\Delta$. Аналогично, через $T_{*}(\mu, \Delta)$ обозначим период цикла из (2.26) релейного уравнения (2.4), для которого, напомним, справедливы асимптотические формулы

$$
T_{*}(\mu, \Delta)=2 \pi-2 \pi \operatorname{ctg}(\Delta) \mu+O\left(\mu^{2}\right), \quad \frac{\partial T_{*}}{\partial \Delta}(\mu, \Delta)=\frac{2 \pi}{\cos ^{2} \Delta} \mu+O\left(\mu^{2}\right),
$$

равномерные по $\Delta$ из любого фиксированного компакта $K \subset(0, \pi)$.

Изучим сначала вопрос о существовании периодического решения с периодом $m \Delta / k$ релейного уравнения (2.4). С этой целью обратимся к аналогичному (1.12) уравнению

$$
T_{*}(\mu, \Delta)=\frac{m \Delta}{k}
$$

и заметим, что в силу (3.1) при $m \geqslant 3$ и любом натуральном

$$
k: 1 \leqslant k<\frac{m}{2}
$$

оно допускает единственное решение $\Delta=\widehat{\Delta}_{(k)}(\mu) \in(0, \pi)$, где

$$
\widehat{\Delta}_{(k)}(\mu)=\frac{2 \pi k}{m}-\frac{2 \pi k}{m} \operatorname{ctg}\left(\frac{2 \pi k}{m}\right) \mu+O\left(\mu^{2}\right) .
$$

Тем самым при $\Delta=\widehat{\Delta}_{(k)}(\mu)$ периодическое решение $(2.26)$ уравнения $(2.4)$ имеет нужный период $m \widehat{\Delta}_{(k)}(\mu) / k$.

Обратимся теперь к периодическому решению $x(t, \varepsilon, \mu, \Delta)$ уравнения $(2.1)$ и рассмотрим соответствующее ему уравнение

$$
T(\varepsilon, \mu, \Delta)=\frac{m \Delta}{k},
$$

считая, что $m \geqslant 3$, а номер $k$ принадлежит множеству (3.3). Напомним, далее, что согласно $(2.27)$ для периода $T(\varepsilon, \mu, \Delta)$ справедливо асимптотическое представление

$$
T(\varepsilon, \mu, \Delta)=T_{*}(\mu, \Delta)+O\left(\varepsilon \mu^{2} \ln \frac{1}{\varepsilon \mu}\right), \quad \varepsilon, \mu \rightarrow 0,
$$


причем, как и в случае (3.1), это представление равномерно по $\Delta$ из любого компакта $K \subset(0, \pi)$. Отсюда и из простоты корня $\Delta=\widehat{\Delta}_{(k)}(\mu)$ уравнения (3.2) очевидным образом следует, что уравнение (3.5) имеет хотя бы один корень $\Delta=\widehat{\Delta}_{(k)}(\varepsilon, \mu)$ с асимптотикой

$$
\widehat{\Delta}_{(k)}(\varepsilon, \mu)=\widehat{\Delta}_{(k)}(\mu)+O\left(\varepsilon \mu^{2} \ln \frac{1}{\varepsilon \mu}\right), \quad \varepsilon, \mu \rightarrow 0 .
$$

Проделанные построения приводят к следующему утверждению.

Теорема 3.1. Для любого натурального $m \geqslant 3$ найдутся такие достаточно малые $\varepsilon_{0}, \mu_{0}>0$, что при всех $0<\varepsilon \leqslant \varepsilon_{0}, 0<\mu \leqslant \mu_{0}$ и при каждом $k$ из множества (3.3) система (1.8) допускает иикл (бегущую волну)

$$
\widehat{C}_{k}: x_{j}=x_{(k)}\left(t+(j-1) \widehat{\Delta}_{(k)}(\varepsilon, \mu), \varepsilon, \mu\right), \quad j=1, \ldots, m,
$$

где $x_{(k)}(t, \varepsilon, \mu)=\left.x(t, \varepsilon, \mu, \Delta)\right|_{\Delta=\widehat{\Delta}_{(k)}(\varepsilon, \mu)}, a \Delta=\widehat{\Delta}_{(k)}(\varepsilon, \mu)-$ коренъ (3.6) уравнения (3.5).

Исследуем теперь вопрос об устойчивости цикла (3.7) с фиксированным номером $k$. В силу леммы 1.2 данный вопрос сводится к анализу расположения корней уравнений

$$
\left(\widehat{\nu}_{s}(\varepsilon, \mu, \varkappa)\right)^{k}=\varkappa^{m}, \quad s \in \mathbb{N},
$$

где $\widehat{\nu}_{s}(\varepsilon, \mu, \varkappa)$ - мультипликаторы $\nu_{s}(\varepsilon, \mu, \varkappa)$ уравнения $(2.53)$ при значении $\Delta=$ $\widehat{\Delta}_{(k)}(\varepsilon, \mu)$ параметра $\Delta$. Впрочем, можно не рассматривать в $(3.8)$ случай $s \geqslant 3$, поскольку, как будет показано далее, полный набор мультипликаторов системы в вариациях

$$
\begin{aligned}
\ddot{h}_{j}+2 \mu \dot{h}_{j}+h_{j} & =\widehat{A}\left(t+(j-1) \widehat{\Delta}_{(k)}(\varepsilon, \mu), \varepsilon, \mu\right) h_{j-1}, \quad j=1, \ldots, m, \\
h_{0} & =h_{m}, \quad \widehat{A}(t, \varepsilon, \mu)=\frac{1}{\varepsilon} F_{x}^{\prime}\left(\frac{x_{(k)}(t, \varepsilon, \mu)}{\varepsilon}\right),
\end{aligned}
$$

на цикле (3.7) строится уже по корням первых двух уравнений (3.8).

Итак, обратимся к уравнениям (3.8) при $s=1,2$, считая, что параметр $\varkappa$ принадлежит шару $B(r)$ при некотором $r>1$. В этом случае согласно теореме 2.2 имеют место асимптотические представления (2.69). Объединяя их с формулами (3.4), (3.6), нетрудно убедиться в том, что при $\varepsilon, \mu \rightarrow 0$ равномерно по $\varkappa \in B(r)$

$$
\widehat{\nu}_{1}(\varepsilon, \mu, \varkappa)=1+\mu \widehat{\lambda}_{+}(\varkappa)+o(\mu), \quad \widehat{\nu}_{2}(\varepsilon, \mu, \varkappa)=1+\mu \widehat{\lambda}_{-}(\varkappa)+o(\mu),
$$

где

$$
\widehat{\lambda}_{ \pm}(\varkappa)=\pi\left[\varkappa-2 \pm \sqrt{(\varkappa-2)^{2}+\frac{4(\varkappa-1)}{\sin ^{2}(2 \pi k / m)}}\right] .
$$

В свою очередь, равенства (3.10), (3.11) позволяют заключить, что при $s=1$ соответствующее уравнение $(3.8)$ имеет корни $\varkappa=\varkappa_{+, l}(\varepsilon, \mu), l=0,1, \ldots, m-1$, 
а при $s=2$ - корни $\varkappa=\varkappa_{-, l}(\varepsilon, \mu), l=0,1, \ldots, m-1$, причем эти корни при $\varepsilon, \mu \rightarrow 0$ допускают асимптотику

$$
\varkappa_{ \pm, l}(\varepsilon, \mu)=\varkappa_{l}\left[1+\mu \frac{k}{m} \widehat{\lambda}_{ \pm}\left(\varkappa_{l}\right)+o(\mu)\right], \quad l=0,1, \ldots, m-1
$$

где $\varkappa_{l}=\exp (2 \pi i l / m)$. Добавим, что в силу леммы 1.2 корням (3.12) соответствует полный набор мультипликаторов $\nu_{ \pm, l}(\varepsilon, \mu), l=0,1, \ldots, m-1$, системы (3.9), где

$$
\begin{gathered}
\nu_{+, l}(\varepsilon, \mu)=\left.\widehat{\nu}_{1}(\varepsilon, \mu, \varkappa)\right|_{\varkappa=\varkappa_{+, l}(\varepsilon, \mu)}, \quad \nu_{-, l}(\varepsilon, \mu)=\left.\widehat{\nu}_{2}(\varepsilon, \mu, \varkappa)\right|_{\varkappa=\varkappa_{-, l}(\varepsilon, \mu)}, \\
l=0,1, \ldots, m-1 .
\end{gathered}
$$

Проанализируем получившиеся равенства (3.13). Опираясь на (2.91), нетрудно заметить, что $\varkappa_{+, 0}(\varepsilon, \mu) \equiv 1, \nu_{+, 0}(\varepsilon, \mu) \equiv 1,\left|\nu_{-, 0}(\varepsilon, \mu)\right|<1$. Для остальных мультипликаторов (3.13) в силу (3.10)-(3.12) при $\varepsilon, \mu \rightarrow 0$ справедливы асимптотические представления

$$
\nu_{ \pm, l}(\varepsilon, \mu)=1+\mu \widehat{\lambda}_{ \pm}\left(\varkappa_{l}\right)+o(\mu), \quad l=1, \ldots, m-1 .
$$

Из соотношений (3.14) следует, что при $\operatorname{Re} \widehat{\lambda}_{ \pm}\left(\varkappa_{l}\right)<0, l=1, \ldots, m-1$, выполняются неравенства $\left|\nu_{ \pm, l}(\varepsilon, \mu)\right|<1, l=1, \ldots, m-1$, а в случае, когда $\operatorname{Re} \widehat{\lambda}_{+}\left(\varkappa_{0}\right)>0$ или $\operatorname{Re} \widehat{\lambda}_{-}\left(\varkappa_{0}\right)>0$ при некотором $l_{0}$, модуль соответствующего мультипликатора (3.14) строго больше единицы. Далее, обратим внимание, что величины $\widehat{\lambda}_{ \pm}\left(\varkappa_{l}\right)$ являются корнями полинома

$$
P(\lambda)=\lambda^{2}+2 \pi\left(2-\varkappa_{l}\right) \lambda+\frac{4 \pi^{2}}{\sin ^{2}(2 \pi k / m)}\left(1-\varkappa_{l}\right),
$$

который оказывается гурвицевым в том и только в том случае, когда

$$
\frac{1}{\sin ^{2}(2 \pi k / m)}<\frac{3(2-\cos (2 \pi l / m))}{2 \cos ^{2}(\pi l / m)} .
$$

Предпринятый выше асимптотический анализ мультипликаторов системы (3.9) позволяет разобраться со свойствами устойчивости циклов (3.7) и получить следующее утверждение.

Теорема 3.2. Предположим, что номер $k$ фиксирован и удовлетворяет требованию (3.3). Тогда найдутся такие достаточно малье $\varepsilon_{0}, \mu_{0}>0$, что при всех $0<\varepsilon \leqslant \varepsilon_{0}, 0<\mu \leqslant \mu_{0}$ и при условии

$$
\frac{1}{\sin ^{2}(2 \pi k / m)}-\frac{3(2-\cos (2 \pi / m))}{2 \cos ^{2}(\pi / m)}<0
$$

цикл $\widehat{C}_{k}$ системь (1.8) экспоненииально орбитально устойчив. Если имеет место строго противоположное (3.17) неравенство, то этот иикл неустойчив. 
ДокАЗАТЕЛЬство. Заметим, что в силу свойства

$$
\frac{3(2-\cos (2 \pi / m))}{2 \cos ^{2}(\pi / m)}=\min _{1 \leqslant l \leqslant m-1} \frac{3(2-\cos (2 \pi l / m))}{2 \cos ^{2}(\pi l / m)}
$$

условие (3.17) влечет за собой выполнение неравенств (3.16) при всех $l=$ $1, \ldots, m-1$. Таким образом, в этом случае имеем

$$
\left|\nu_{ \pm, l}(\varepsilon, \mu)\right|<1, \quad l=1, \ldots, m-1,
$$

а значит, интересующий нас цикл (3.7) экспоненциально орбитально устойчив. Если, напротив, выполнено строго противоположное (3.17) неравенство, то заведомо найдется такое $l=l_{0}, 1 \leqslant l_{0} \leqslant m-1$, при котором полином (3.15) допускает корень с положительной действительной частью. Тогда модуль соответствующего мультипликатора (3.14) строго больше единицы и, следовательно, цикл $\widehat{C}_{k}$ системы (1.8) оказывается неустойчивым.

Отдельного рассмотрения заслуживает вопрос о сосуществовании в системе (1.8) устойчивых циклов (3.7) с различными номерами $k$. Для того чтобы сформулировать соответствующий результат, фиксируем произвольно постоянные $c_{1}, c_{2}$, удовлетворяющие оценкам

$$
\arcsin \sqrt{\frac{2}{3}} \leqslant c_{1}<c_{2} \leqslant \pi-\arcsin \sqrt{\frac{2}{3}} .
$$

Теорема 3.3. При всех достаточно малых независимых $\varepsilon>0 u \mu>0$ в системе (1.8) сосуществуют экспоненциально орбитально устойчивые ииклы (3.7) с номерами

$$
c_{1} \leqslant \frac{2 \pi k}{m} \leqslant c_{2}
$$

ДокАзАТЕЛьСтво вытекает из очевидного неравенства

$$
\frac{3(2-\cos (2 \pi / m))}{2 \cos ^{2}(\pi / m)}>\frac{3}{2} .
$$

Действительно, в силу выбора постоянных $c_{1}, c_{2}$ (см. (3.18)) для всех номеров $k$ из множества (3.19) имеем $\sin ^{2}(2 \pi k / m) \geqslant 2 / 3$. Отсюда и из $(3.20)$, в свою очередь, следует, что для каждого из таких $k$ выполняется условие устойчивости (3.17).

Добавим, кроме того, что при $m \rightarrow \infty$ количество номеров $k$, для которых справедливы требования (3.19), неограниченно растет. Это значит, что при подходящем увеличении $m$ и уменьшении параметров $\varepsilon, \mu$ можно добиться сосуществования в системе (1.8) любого наперед заданного конечного числа устойчивых циклов. Таким образом, установлена реализуемость в рассматриваемой задаче известного феномена буферности.

3.2. Заключение. Отметим, что предложенные нами методы исследования периодических решений типа бегущих волн можно применять не только для кольцевых цепочек однонаправленно связанных обыкновенных дифференциальных уравнений. Как оказывается, они распространяются и на цепочки уравнений с запаздыванием вида

$$
\dot{x}_{j}=f\left(x_{j}, x_{j}\left(t-h_{1}\right), x_{j-1}, x_{j-1}\left(t-h_{2}\right)\right), \quad j=1, \ldots, m, \quad x_{0}=x_{m},
$$


где $m \geqslant 2, x_{j}=x_{j}(t) \in \mathbb{R}^{n}, h_{1}, h_{2}>0$, а вектор-функция $f(x, y, z, v)$ со значениями в $\mathbb{R}^{n}$ бесконечно дифференцируема по $(x, y, z, v) \in \mathbb{R}^{4 n}$.

Примером цепочки (3.21) служит математическая модель кольцевой нейронной сети Хопфилда с запаздыванием, представляющая собой систему

$$
\dot{u}_{j}=-\mu u_{j}+\lambda\left[1-(a+1) f\left(u_{j}(t-1)\right)-b g\left(u_{j-1}\right)\right], \quad j=1, \ldots, m .
$$

Здесь $u_{0}=u_{m}, \mu, a, b=$ const $>0, \lambda \gg 1$, а для функций $f(u), g(u) \in C^{\infty}(\mathbb{R})$ справедливы аналогичные (1.7) асимптотические представления

$$
\begin{aligned}
& f(u)= \begin{cases}\sum_{l=1}^{\infty} \frac{c_{l}^{-}}{u^{l}} & \text { при } u \rightarrow-\infty, \\
1+\sum_{l=1}^{\infty} \frac{c_{l}^{+}}{u^{l}} & \text { при } u \rightarrow+\infty,\end{cases} \\
& g(u)= \begin{cases}\sum_{l=1}^{\infty} \frac{d_{l}^{-}}{u^{l}} & \text { при } u \rightarrow-\infty, \\
1+\sum_{l=1}^{\infty} \frac{d_{l}^{+}}{u^{l}} & \text { при } u \rightarrow+\infty,\end{cases}
\end{aligned}
$$

сохраняющие силу при дифференцировании по $и$ любое число раз.

Другой пример - математическая модель кольцевой нейронной сети с однонаправленными химическими связями, имеющая вид

$$
\dot{u}_{j}=\left[\lambda f\left(u_{j}(t-1)\right)+b g\left(u_{j-1}\right) \ln \frac{u_{*}}{u_{j}}\right] u_{j}, \quad j=1, \ldots, m, \quad u_{0}=u_{m} .
$$

Здесь $\lambda \gg 1, b=$ const $>0, u_{*}=\exp (c \lambda), c=$ const $\in \mathbb{R}$, а функции $f(u)$, $g(u) \in C^{2}\left(\mathbb{R}_{+}\right), \mathbb{R}_{+}=\{u \in \mathbb{R}: u \geqslant 0\}$, обладают следующими свойствами:

$$
\begin{gathered}
f(0)=1 ; \quad f(u)+a, u f^{\prime}(u), u^{2} f^{\prime \prime}(u)=O\left(\frac{1}{u}\right) \quad \text { при } u \rightarrow+\infty ; \\
g(u)>0 \quad \forall u>0, \quad g(0)=0 ; \\
g(u)-1, u g^{\prime}(u), u^{2} g^{\prime \prime}(u)=O\left(\frac{1}{u}\right) \quad \text { при } u \rightarrow+\infty,
\end{gathered}
$$

где $a=$ const $>0$.

Для системы (3.22) подробный анализ вопросов о существовании и устойчивости бегущих волн вида (1.13) в простейшем случае $k=1$ предпринят в работе [5], а для системы (3.23) авторы планируют отдельную статью.

Отметим еще один интересный факт. Из наших построений вытекает, что в случае

$$
\varepsilon=1, \quad 0<\mu \ll 1
$$

утверждения, содержащиеся в теореме 2.1, лемме 2.1, лемме 2.2 и следствиях $2.1,2.2$, сохраняются дословно, а в теореме 2.2 формулы (2.69) заменяются на аналогичные равенства с остатками $O(\mu)$. Связано это с тем, что фигурирующие в указанных остатках слагаемые порядка $\sqrt{\varepsilon} \mu$ при $\varepsilon=1$ становятся величинами порядка $\mu$. 
Из сказанного чуть выше следует, что при условиях (3.24) утверждение теоремы 3.1 о существовании у системы (1.8) циклов (3.7) заведомо остается в силе. Вопрос об устойчивости этих циклов несколько сложнее. Проблема здесь заключается в том, что при обосновании теоремы 3.2 мы уже не вправе пользоваться аналогами равенств (2.69), так как теперь остатки в них, имеющие порядок $O(\mu)$, могут повлиять на свойства устойчивости рассматриваемых циклов.

Для решения сформулированной проблемы попытаемся провести доказательство теоремы 3.2, опираясь на асимптотические равенства (2.90), остатки в которых при $\varepsilon=1$ приобретают порядок $O\left(\mu^{3 / 2}\right)$. Поскольку формулы (2.90) справедливы при $\varkappa \in B_{\delta}(r)$, мы a priori должны предполагать, что все корни (3.12) уравнений (3.8) при $s=1,2$ принадлежат множеству $B_{\delta}(r)$ при некоторых $r>1, \delta>0$. В свою очередь, для этого достаточно убедиться в том, что

$$
\left\{\varkappa \in \mathbb{C}:\left|\varkappa-\varkappa_{j, *}(\varepsilon, \mu)\right| \leqslant \delta\right\} \cap\{\varkappa \in \mathbb{C}:|\varkappa|=1\}=\varnothing, \quad j=1,2,
$$

где $\varkappa_{j, *}(\varepsilon, \mu), j=1,2,-$ функции (2.88).

Проанализируем требования (3.25). Нетрудно увидеть, что в силу вещественности корней $\varkappa_{*, j}^{0}, j=1,2$, уравнения (2.89) проверка этих условий сводится к проверке выполнения неравенств $\varkappa_{*, j}^{0} \neq \pm 1, j=1,2$, которые имеют место при $\Delta \neq \Delta_{j}^{0}, j=1,2$, где

$$
\Delta_{1}^{0}=\arcsin \frac{\sqrt{8}}{3}, \quad \Delta_{2}^{0}=\pi-\arcsin \frac{\sqrt{8}}{3} .
$$

Итак, для справедливости условий (3.25) при подходящем уменьшении $\delta$ параметр $\Delta$ должен быть фиксирован и отличен от критических величин (3.26). Напомним, далее, что на самом деле нас интересуют только дискретные значения $\Delta$, задающиеся равенствами (3.6). Принимая во внимание это обстоятельство и учитывая неравенства

$$
\frac{2 \pi k}{m} \neq \Delta_{j}^{0}, \quad j=1,2
$$

(которые будут установлены отдельно), получаем следующий результат.

Теорема 3.4. В случае (3.24) для всех ииклов (3.7) сохраняются утверждения теоремы 3.2 об устойчивости или неустойчивости.

Для придания сформулированной теореме необходимой строгости осталось убедиться в выполнении требований (3.27) при всех интересующих нас номеpax $m$ и $k$. В связи с этим обратим внимание, что указанные требования заведомо имеют место при

$$
(1 \pm i \sqrt{8})^{m} \neq 3^{m} .
$$

В свою очередь, для проверки выполнения соотношений (3.28) достаточно показать, что

$$
y_{m} \stackrel{\text { def }}{=} \operatorname{Re}\left[(1+i \sqrt{8})^{m}\right] \neq 0 \quad(\bmod 3) \quad \forall m \geqslant 1 .
$$

Свойства (3.29) нетрудно установить с помощью метода математической индукции. Действительно, при $m=1,2$ они очевидны, так как $y_{1}=1, y_{2}=-7$. 
Предположим, далее, что $y_{s} \neq 0(\bmod 3)$ при $s=1, \ldots, m+1$, и убедимся в том, что $y_{m+2} \neq 0(\bmod 3)$. Для этого рассмотрим рекуррентное соотношение

$$
y_{m+2}=2 y_{m+1}-9 y_{m},
$$

справедливое для последовательности $y_{m}, m \geqslant 1$. Из (3.30) очевидным образом имеем $y_{m+2}=2 y_{m+1}(\bmod 3)$. Отсюда и из предполагаемого неравенства $y_{m+1} \neq 0(\bmod 3)$ вытекает, что $y_{m+2} \neq 0(\bmod 3)$. Таким образом, неравенства (3.29) полностью обоснованы.

Следует отметить, что утверждения теорем 3.1, 3.2 сохраняются, естественно, и в более общем случае $\varepsilon=\varepsilon_{0}, \varepsilon_{0}=$ const $>0,0<\mu \ll 1$. Упомянутый случай сводится к уже разобранной ситуации (3.24) посредством нормировки $\varepsilon / \varepsilon_{0} \rightarrow \varepsilon$ и переобозначения $F\left(x / \varepsilon_{0}\right) \rightarrow F(x)$.

Отдельного упоминания заслуживает следующая нерешенная проблема, связанная с уравнением (2.1). В настоящей работе это уравнение использовалось как вспомогательное, однако оно представляет и самостоятельный интерес, поскольку согласно [1] является моделью одноконтурного RCL-генератора с запаздыванием в цепи обратной связи.

Выше нам удалось показать (см. теоремы 2.1, 2.2 и следствия $2.1,2.2$ ), что в случае, когда запаздывание $\Delta$ принадлежит интервалу (2.2), уравнение $(2.1)$ допускает экспоненциально орбитально устойчивый цикл, но при $\Delta>\pi$ вопрос об аттракторах этого уравнения пока не изучен.

В заключение работы представим результаты компьютерного анализа системы (1.8) при

$$
F(x)=-\frac{x}{\sqrt{x^{2}+1}}, \quad \varepsilon=0.1, \quad \mu=0.005, \quad m=9,11,15 .
$$

Численное интегрирование этой системы проводилось методом Рунге-Кутта четвертого порядка точности с постоянным шагом $10^{-4}$ и начальными условиями

$$
\begin{gathered}
\left.x_{j}\right|_{t=0}=\frac{2}{\pi \mu} \sin \left(\frac{2 \pi k}{m}\right) \sin \left((j-1) \frac{2 \pi k}{m}\right), \\
\left.\dot{x}_{j}\right|_{t=0}=\frac{2}{\pi \mu} \sin \left(\frac{2 \pi k}{m}\right) \cos \left((j-1) \frac{2 \pi k}{m}\right), \quad j=1, \ldots, m, \quad 1 \leqslant k<\frac{m}{2},
\end{gathered}
$$

которые в первом приближении соответствуют начальным условиям интересующих нас циклов (3.7). Было установлено следующее:

1) при $m=9$ циклы $\widehat{C}_{k}, k=1,4$, неустойчивы, а $\widehat{C}_{k}, k=2,3$, устойчивы;

2) при $m=11$ циклы $\widehat{C}_{k}, k=1,5$, неустойчивы, а $\widehat{C}_{k}, k=2,3,4$, устойчивы;

3) при $m=15$ циклы $\widehat{C}_{k}, k=1,6,7$, неустойчивы, а $\widehat{C}_{k}, k=2,3,4,5$, устойчивы.

В случае $m=9$ проекции устойчивых циклов $\widehat{C}_{2}, \widehat{C}_{3}$ на плоскость $\left(x_{1}, x_{2}\right)$ представлены на рис. 4, 5 соответственно.

Полученные свойства устойчивости полностью согласуются с теоретическими результатами, содержащимися в теореме 3.2. Таким образом, предпринятый нами асимптотический анализ заведомо применим в случае (3.31). Добавим еще, что при дальнейшем уменьшении $\mu$ существенно возрастает время "выхода" системы на тот или иной устойчивый периодический режим, что затрудняет интерпретацию численных экспериментов. 




Рис. 4

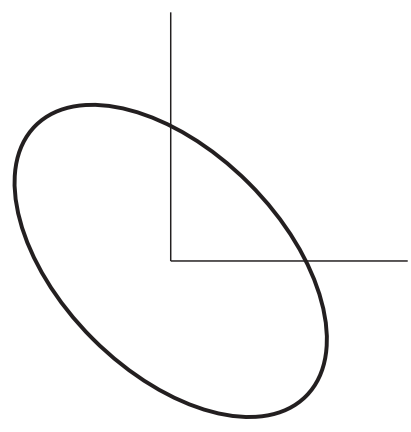

Рис. 5

Авторы выражают глубокую признательность рецензенту за внимательное ознакомление с рукописью и высказанные полезные замечания и соображения, способствовавшие существенному улучшению содержания статьи.

\section{Список литературы}

1. А. Ю. Колесов, Е.Ф. Мищенко, Н. Х. Розов, "Новые методы доказательства существования и устойчивости периодических решений в сингулярно возмущенных системах с запаздыванием", Анализ и особенности. Часть 2, Сборник статей. K 70-летию со дня рождения академика Владимира Игоревича Арнольда, Тр. МИАН, 259, Наука, М., 2007, 106-133; англ. пер.: А. Yu. Kolesov, E. F. Mishchenko, N. Kh. Rozov, "New methods for proving the existence and stability of periodic solutions in singularly perturbed delay systems", Proc. Steklov Inst. Math., 259 (2007), $101-127$.

2. С. П. Стрелков, Введение в теорию колебаний, 2-е изд., Наука, М., 1964, 437 с.

3. А. С. Дмитриев, А. И. Панас, Динамический хаос: новые носители информации для систем связи, Физматлит, М., 2002, 252 с.

4. Д.К. Спротт, Элегантный хаос: алгебраччески простые хаотические потоки, Ижевский институт компьютерных исследований, М.-Ижевск, 2012, 328 с.; пер. с англ.: J. C. Sprott, Elegant chaos. Algebraically simple chaotic flows, World Scientific Publishing Co. Pte. Ltd., Hackensack, NJ, 2010, xvi+285 pp.

5. С. Д. Глызин, А. Ю. Колесов, Н. Х. Розов, "Релаксационные автоколебания в сетях Хопфилда с запаздыванием", Изв. РАН. Сер. матем., 77:2 (2013), 53-96; англ. пер.: S. D. Glyzin, A. Yu. Kolesov, N. Kh. Rozov, "Relaxation self-oscillations in Hopfield networks with delay", Izv. Math., 77:2 (2013), 271-312.

6. С. Д. Глызин, А. Ю. Колесов, Н.Х. Розов, "О явлениях хаоса в кольце из трех однонаправленно связанных генераторов", Ж. вычисл. матем. и матем. физ., 46:10 (2006), 1809-1821; англ. пер.: S. D. Glyzin, A. Yu. Kolesov, N. Kh. Rozov, "Chaos phenomena in a circle of three unidirectionally connected oscillators", Comput. Math. Math. Phys., 46:10 (2006), 1724-1736.

7. T. Kapitaniak, L. O. Chua, "Hyperchaotic attractors of unidirectionally-coupled Chua's circuits", Internat. J. Bifur. Chaos Appl. Sci. Engrg., 4:2 (1994), 477-482.

8. I. P. Mariño, V. Pérez-Muñuzuri, V. Pérez-Villar, E. Sánchez, M. A. Matías, "Interaction of chaotic rotating waves in coupled rings of chaotic cells", Phys. D, 128:2-4 (1999), 224-235. 
9. P. Perlikowski, S. Yanchuk, M. Wolfrum, A. Stefanski, P. Mosiolek, T. Kapitaniak, "Routes to complex dynamics in a ring of unidirectionally coupled systems", Chaos, 20:1 (2010), 1-10.

10. А.Ю. Колесов, Е.Ф. Мищенко, Н.Х. Розов, "Реле с запаздыванием и его $C^{1}$-аппроксимация", Динамические системы и смежные вопросы, Сб. статей. K 60-летию со дня рождения академика Дмитрия Викторовича Аносова, Тр. МИАН, 216, Наука, М., 1997, 126-153; англ. пер.: А. Yu. Kolesov, E. F. Mishchenko, N. Kh. Rozov, "Relay with delay and its $C^{1}$-approximation", Proc. Steklov Inst. Math., 216 (1997), 119-146.

11. А. Ю. Колесов, Е.Ф. Мищенко, Н. Х. Розов, "Об одной модификации уравнения Хатчинсона", Ж. вычисл. матем. и матем. физ., 50:12 (2010), 2099-2112; англ. пер.: A. Yu. Kolesov, E. F. Mishchenko, N. Kh. Rozov, "A modification of Hutchinson's equation", Comput. Math. Math. Phys., 50:12 (2010), 1990-2002.

12. С. Д. Глызин, А. Ю. Колесов, Н. Х. Розов, "Релаксационные автоколебания в нейронных системах. I", Дифферени. уравнения, 47:7 (2011), 919-932; англ. пер.: S. D. Glyzin, A. Yu. Kolesov, N. Kh. Rozov,, "Relaxation self-oscillations in neuron systems. I", Differ. Equ., 47:7 (2011), 927-941.

СЕРГЕЙ ДМИТРИЕВИч ГЛЫЗИН

Поступило в редакцию

(SERgey D. Glyzin)

12.02 .2013

Ярославский государственный университет

21.07 .2013

им. П. Г. Демидова

E-mail: glyzin@uniyar.ac.ru

АндРей Юрьевич КоЛЕСов

(ANdrei Yu. Kolesov)

Ярославский государственный университет

им. П. Г. Демидова

E-mail: kolesov@uniyar.ac.ru

НиколАй Христович Розов

(Nikolai Kh. Rozov)

Московский государственный университет

им. М. В. Ломоносова

E-mail: fpo.mgu@mail.ru 